\title{
Vers l'optoélectronique organique bas coût
}

\author{
Jean-Pierre Moliton et Thierry Trigaud \\ XLIM/CNRS, Pôle Limousin des Sciences et Technologies de l'Information et de la \\ Communication, Université de Limoges, 123 avenue A. Thomas, 87060 Limoges cedex, France.
}

\begin{abstract}
Résumé - A travers quelques exemples de réalisations de composants d'un réseau local de communications optiques, est dressé un court panel non exhaustif des possibilités offertes par la matière organique dans le secteur des micro et nanotechnologies. Sont passés en revue les avantages et les inconvénients des milieux organiques et des dispositifs résultants, tout en s'attardant sur les spécificités des procédés de la filière organique, notamment dans le cas de la fabrication de diodes électroluminescentes et de dispositifs à bande interdite photonique.
\end{abstract}

Mots-clefs - OLEDs, PLEDs, BIP, polymères conducteurs, petites molécules.

\section{I- LA VOIE ORGANIQUE ; LES RAISONS D'ESPERER}

\section{A. A quand l'âge de la maturité pour les dispositifs organiques?}

Si les propriétés semi-conductrices des matériaux organiques sont connues depuis le début des années 60, on ne peut pas dire que leur usage dans des systèmes ou même simplement des composants électroniques ou optoélectroniques ait atteint le stade de la production industrielle de grande série, signe d'une maturité certaine. Que la société Eastman Kodak propose à l'achat sur son site internet des barrettes de photodiodes pour écran organique, que la société japonaise Pioneer, sur cette base, ait commercialisé depuis 1997 des autoradios présentant en façade de tels écrans, que CDT (Cambridge Display Technologies) ait signé des accords de licence avec Philips depuis 1992 et encore en 2004 avec Thales Avionics LCD, constituent autant d'indications encourageantes sans cependant être les preuves intangibles de cette maturité. L'installation depuis une petite dizaine d'années par Philips d'une ligne pilote de production de diodes électroluminescentes polymères (PLEDs) à Heerlen, sans l'existence d'un système commercial de distribution, semble indiquer au contraire que des difficultés existent. Jeremy Burroughes, le Directeur technique de CDT, s'inquiétait d'ailleurs récemment de ne pouvoir observer que l'existence potentielle, et non réelle, de débouchés commerciaux (1), alors que sur le plan technologique les PLEDs apparaissent vraiment abouties, et très largement compétitives en performances. C'est sans doute que ces critères sont insuffisants dès lors que l'on s'attaque à des marchés existants où d'autres dispositifs équivalents occupent depuis longtemps la place avec succès et ont déjà fait leurs preuves aux yeux du consommateur. La même constatation est vraie dans le secteur du développement des dispositifs photovoltaïques où les filières silicium s'accaparent la part majoritaire du financement. Même s'il est courant de penser que des niches d'exploitation existent pour les systèmes organiques, l'opinion générale est de considérer que les dispositifs organiques doivent offrir autre chose en plus, des fonctionnalités nouvelles, ou des économies en moyens technologiques de production industrielle.

C'est à ce titre que sont apparues des idées de procédés nouveaux de fabrication pour les dispositifs organiques. Toutes visent à supprimer l'usage des salles blanches au niveau de la production, en raison d'un coût prohibitif de construction et surtout de maintenance. Les industriels habituellement proches des matériaux plastiques appréhendent en effet l'introduction onéreuse des technologies en salle blanche, alors que ceux qui en font un usage courant ne veulent en aucun cas les polluer par la mise en œuvre de matériaux organiques, étant donné que leurs fabrications à base de semiconducteurs classiques font presque ou pratiquement aussi bien. Que ce soit l'idée d'une fabrication en "tuyau", ou celle de faire appel aux lithographies douces (ou "soft lithographies"), ces deux nouvelles grandes familles de procédés donnent naissance en amont à de nouvelles recherches à caractère fondamental. Le point commun à ces études est de les rapprocher de l'électronique moléculaire, domaine qu'il était jusqu'alors convenu de considérer comme uniquement prospectif. En effet, parmi les objectifs poursuivis en optoélectronique organique, ainsi qu'on le verra un peu plus loin, se trouve l'abaissement de la consommation entraînant celle des tensions de polarisation, et par voie de conséquence une diminution des épaisseurs des couches mises en jeu, qui dans un grand nombre de cas ne dépassent pas $50 \mathrm{~nm}$. L'électronique et l'optoélectronique organiques tendent ainsi à diminuer les effets de volume de la matière organique au profit de ceux d'interface, que ce soit entre films organiques eux-mêmes ou aux contacts métal milieu organique. Il est d'ailleurs symptomatique de constater de ce point de vue que le dernier volume de la revue Materialstoday est entièrement consacré à l'énergétique de ces interfaces (2). Un nombre considérable de travaux est désormais engagé à travers le monde, USA, Allemagne et Japon notamment. Des sociétés disposant de capitaux importants, comme Sandia 
Corp., Lockheed Martin Comp., et l'US Department of Energy's National Nuclear Security Administration, ont lancé des programmes communs de recherche dans le domaine.

Comme conséquence essentielle de cette tendance, on notera le creusement du fossé entre la science et les technologies de l'électronique des semiconducteurs minéraux classiques, au sens large, et celles des milieux organiques. Ainsi se dirige-t-on vers une situation de rupture technologique où les fondeurs qui ne se seront pas investis dans les technologies organiques ne pourront pas espérer une reconversion, même au prix de cessions de licence tant la spécificité du secteur est grande. Si l'âge de maturité de l'électronique et de l'optoélectronique organique n'est pas encore atteint, il approche à grand pas. A titre d'exemple, on examinera dans le paragraphe suivant ce qu'il est possible dès maintenant d'envisager avec la matière organique dans un réseau local de communications.

\section{B. Exemple d'un réseau local de communication "tout organique".}

L'aperçu donné par le schéma de la figure 1 résume les pièces essentielles d'un tel réseau. Il fournit plus généralement un échantillonnage assez représentatif des composants que l'on peut espérer fabriquer en matériau organique.

Il est clair que ce réseau est de type communication optique, et c'est une des forces des filières organiques. On sait bien en effet que les besoins en quantité d'informations transportées nécessitent des fréquences de signaux de plus en plus élevées et des largeurs de bande passante toujours de plus en plus grandes. Les vitesses de commutation et la précision de synchronisation des signaux nécessaires font que les circuits électroniques en semiconducteurs classiques, silicium, GaAs, InSb, InP ou tout autre composé III-V, éprouvent des difficultés à évacuer la puissance dissipée. A titre d'exemple, on envisage d'enfermer ces circuits dans des baies réfrigérées à $-40^{\circ} \mathrm{C}$. C'est la raison pour laquelle les photons sont de plus en plus préférés aux électrons. De ce point de vue, les milieux organiques sont des candidats de choix, puisqu'ils sont très souvent transparents à la lumière, tout au moins dans le visible. Pour les longueurs d'onde retenues pour les télécommunications, $1,3 \mu \mathrm{m}$ et $1,55 \mu \mathrm{m}$, il faut être par contre attentif dans le choix du milieu organique envisagé, étant donné que ces longueurs d'onde peuvent se situer dans un des domaines d'absorption des fréquences de vibration d'élongation $v(\mathrm{C}-\mathrm{H})$, et que la liaison $\mathrm{C}-\mathrm{H}$ est à la base de la matière organique. A titre d'exemple, le fondamental de cette vibration dans le PMMA est à 3,3 $\mu \mathrm{m}$. Envisageons maintenant successivement chacun des éléments constituant le réseau et les potentialités de la voie organique.

Les sources organiques de lumière existent déjà, et sont même assez répandues, ce sont les diodes organiques électroluminescentes (OLEDs); elles constituent une des plus belles réalisations de la voie organique (cf. le § III.1). La pureté spectrale nécessaire à un réseau de communication nécessite toutefois d'enfermer le milieu luminescent dans une microcavité. Si le laser tout organique pompé électriquement n'est pas encore envisageable dans l'immédiat, des microcavités lasers pompées optiquement ont déjà été fabriquées ou sont en cours d'étude avancée. On rencontre en gros trois types de technologie :

les microcavités constituées de deux réflecteurs, dont un au moins est filtrant, en général un réflecteur de Bragg. Le réseau de Bragg est souvent photo inscrit dans le plan du guide comme dans un laser DFB (Distributed Feedback Laser); les longueurs d'onde actuelles d'émission sont respectivement de : $604 \mathrm{~nm}$ (3) et 609 $\mathrm{nm}(4)$.

les microcavités à mode de galerie constituées d'une cavité en anneaux ( $60 \mu \mathrm{m}$ de diamètre) de polymère dopé le long d'une fibre. L'émission peut être en géométrie transverse ou longitudinale en régime multimode ou monomode. Pour l'instant, l'onde de pompe se situe à $532 \mathrm{~nm}$ (4).

Les microcavités planaires, plus délicates à fabriquer, qui s'interprètent également en mode de galerie, avec des géométries toriques ou granulaires (4).

Le milieu amplificateur placé dans la cavité est en général un polymère dopé, par exemple au DCM (4dicyanmethylène-2-methyl-6-4H-pyrane) (3). Mais bien d'autres existent (16) Des seuils de densité d'énergie de $400 \mu \mathrm{J} / \mathrm{cm}^{2}$ avec des gains efficaces au seuil de $250 \mathrm{~cm}^{-1}$ ont pu être obtenus dans le premier cas de structure, le plus simple à réaliser (3).

Le modulateur du signal électrique adopte jusqu'alors la structure d'un interféromètre de type Mach-Zehnder, en mettant en œuvre une structure planaire optiquement non linéaire, selon le schéma de la figure 2. Le signal électrique module par effet électro-optique l'indice d'un des deux bras de l'interféromètre sur la longueur des électrodes, alors que l'autre branche sert de référence.

Le paramètre critique est la tension de retournement $\mathrm{V}_{\pi}$ pour laquelle le signal ayant transité par le bras actif est déphasé de $\pi$ par rapport à celui passé par le bras de référence. Son expression est donnée par: $\mathrm{V}_{\pi}=\frac{\lambda \mathrm{d}}{\mathrm{n}_{\mathrm{e}}^{2} \mathrm{r}_{33} \mathrm{~L}}$ où $\lambda$ est la longueur d'onde du signal, d la distance entre électrodes, L la longueur des électrodes, $\mathrm{n}_{\mathrm{e}}$ l'indice effectif du milieu électro-optique, et $r_{33}$ le coefficient électro-optique. C'est à ce niveau qu'on rencontre une des difficultés par rapport à laquelle des progrès sont nécessaires. Les valeurs de $\mathrm{V}_{\pi}$ sont en effet en général trop élevées pour des longueurs de bras de l'ordre de grandeur d'une microstructure. A titre d'exemple, avec un groupement chromophore de DR1 mélangé à un polyimide fluoré (6FDA-ODA) pour lequel $\mathrm{r}_{33}=10 \mathrm{pm} / \mathrm{V}$, une longueur $\mathrm{L}=1,8 \mathrm{~cm}$ est nécessaire avec un $\mathrm{V}_{\pi}=180 \mathrm{~V}$ (5). Récemment ont été synthétisés des 
matériaux organiques dont le $\mathrm{r}_{33}$ dépasse $180 \mathrm{pm} / \mathrm{V}$; mais ce ne sont que des spécimens de laboratoire de chimie produits en trop petite quantité pour envisager la production d'un composant (6).

Une autre difficulté réside dans l'efficacité de modulation des électrodes le long du bras du modulateur. Plusieurs possibilités sont offertes; quelle que soit la méthode choisie, l'idéal consiste à trouver un milieu organique électro-optique dont la permittivité électrique à la fréquence micro-onde soit très inférieure à celle de la fréquence optique de manière à obtenir pour les deux fréquences des vitesses de phase sensiblement équivalentes (7). Cet accord d'indice est difficile à réaliser, sans parler des complications causées par la mesure elle-même des permittivités électriques dans la gamme souhaitée [20 - 200] Ghz., pour laquelle des études sont encore indispensables.

Les fibres optiques organiques existent depuis une quinzaine d'années et sont en usage courant partout où de faibles rayons de courbure s'imposent et excluent la fibre classique $\mathrm{SiO}_{2}$. En raison de l'atténuation résultant des effets d'absorption signalés précédemment, des longueurs supérieures à $40 \mathrm{~m}$ ne sont pas envisageables. Dans le même ordre d'idées, des guides plans sont faisables facilement par des procédés secs de gravure, tels que la gravure classique RIE (Reactive Ion Etching) (8) ou d'autres (9), dont les faisceaux d'ions focalisés qui permettent d'obtenir des flancs lisses limitant les pertes optiques (10). PMMA, CR39 et les dérivés poly(amidoimides) se révèlent dans ce cas les meilleurs candidats comme matériaux.

La démodulation et le tri en longueurs d'ondes sont envisageables par le procédé WDM (Wavelength Division Multiplexing) en utilisant à la source une modulation en longueurs d'onde. La puissance de traitement optique de l'information de cette méthode est en effet séduisant; rappelons simplement à cet effet qu'une modulation à $250 \mathrm{GHz}$ à la longueur d'onde de $1,55 \mu \mathrm{m}$ permet de passer théoriquement 10 canaux séparés de seulement $2 \mathrm{~nm}$, avec par conséquent une largeur de bande passante de $20 \mathrm{~nm}$. Une façon astucieuse d'y parvenir est de mettre en œuvre un cristal photonique 2D directement gravé sur le guide plan. La difficulté résulte dans la gravure de ce réseau; des dimensions sub-microniques sont en effet nécessaires. Le $\S$ III.2 fait le point sur ce sujet.

La photodiode de détection s'apparente au plan de la réalisation aux jonctions classiques qui ont été les premières réalisations effectuées en matériau organique (11-13). Des perfectionnements sont sans doute nécessaires pour affiner les temps de commutation, mais il n'existe pas à priori à notre connaissance de difficultés majeures à ce niveau. Ce type de composant devrait en outre bénéficier des progrès technologiques effectués dans le secteur du photovoltaïque, et des LEDs.

Les écrans de réception font partie des réalisations les plus en pointe de l'électronique organique. La page électronique souple a fait la une de plus d'un éditorial dans la presse, même non scientifique. La spécificité des matériaux organiques par rapport aux minéraux est en effet d'être flexibles et, par là, de pouvoir se prêter à un emploi nomade. Un grand nombre de sociétés, parmi lesquelles citons sans avoir la prétention d'être exhaustif, Kodak, Epson, Seïk, Agfa, Pioneer, Philips, Sharp, CDT, Canon, Osram/Siemens- Infineon, DAI Nippon Printing, Universal Display Corp., ont toutes développé des écrans organiques, fonctionnant sur la base de composants variés, dont le type majoritaire reste la diode électroluminescente. Il n'en reste pas moins vrai que pour actionner ces dispositifs d'affichage, il est fait appel dans la plupart des cas à des TFT en silicium amorphe. On touche là une des faiblesses de la voie organique; le transistor organique n'est pas particulièrement performant au plan des vitesses de commutation et de la puissance dissipée. La commande d'un pixel ne nécessite toutefois pas une grande rapidité, et il n'est pas absurde par ailleurs d'envisager un transistor organique transparent. C'est dans ce sens que sont orientées les études actuelles sur les transistors organiques.

Avant d'entrer plus avant dans certains détails de fonctionnement de composants de base qui interviennent dans un des dispositifs que nous venons de présenter, et d'entrevoir par ce biais les voies ouvertes pour progresser, il est essentiel de bien maîtriser quelques aspects propres à la matière organique.

\section{II- ELEMENTS DE PHYSIQUE ELECTRONIQUE DE LA MATIERE ORGANIQUE}

\section{A. Différents types de matériaux organiques.}

Il n'est pas dans notre intention d'effectuer une présentation exhaustive (cf. ref. 16) qui n'aurait pas sa place ici, mais simplement de clarifier la situation en rappelant quelques propriétés de base, et en citant des éléments de vocabulaire. Les milieux organiques peuvent être répertoriés suivant plusieurs modes de classement, par exemple suivant leurs usages, ou suivant leur conductivité électrique.

a- Suivant leurs usages, il est possible de distinguer :

les composites, maintenant abandonnés en micro et nanotechnologies,

les petites molécules, telles que $\mathrm{Alq}_{\mathrm{x}}$, mises en œuvre essentiellement dans les LEDs,

les oligomères, molécules à petit nombre de monomères (< quelques centaines) dans les LEDs, et les transistors,

les polymères, parmi lesquels on différencie :

les polymères classiques qui peuvent être utilisés en tant qu'isolant, comme guides optiques, ou en connectique après avoir été rendus conducteurs par bombardement ionique $(14,15)$,

Les polymères conjugués (polymères présentant en alternance une simple et une double liaison), qu'on rencontre dans les LEDs, les transistors, et les cellules 
photovoltaïques. Ils sont souvent improprement appelés "polymères conducteurs", objets du prix Nobel de chimie de l'année 2000 (Alan Mac Diarmid, Alan Heeger, et Hideki Shirakawa) $(18,19)$,

Les polymères non-linéaires optiques, que l'on rencontre dans les modulateurs électro-optiques, et les lasers.

les organisations supra-moléculaires, qui sont constituées de molécules spatialement ordonnées par la maîtrise des énergies de surface.

b- Suivant la valeur de la conductivité électrique, et en référence aux matériaux classiques, l'échelle de la figure 3 livre des indications intéressantes qui appellent en même temps quelques commentaires.

Si le polyacéthylène dopé à l'iode atteint la conductivité du cuivre, et mérite effectivement l'appellation de "métal organique", non seulement en référence à cette valeur de la conductivité, mais également par rapport au mode de conduction qui est dans le polyacéthylène en général effectivement de nature semblable à celle des métaux (17), ce matériau n'est pas stable à l'air libre. Comme la plupart des polymères conjugués, il s'oxyde dans cette situation et perd ses propriétés métalliques. Sa conductivité non seulement chute alors à une valeur inférieure au S. $\mathrm{cm}^{-1}$, dépendant de son degré d'oxydation, mais aussi change de mécanisme en passant du type courant de dérive (souvent dit " drift") au modèle par saut ("hopping"). Ce dernier est dans les conditions normales le mode de conduction de tous les polymères. C'est la raison pour laquelle les mobilités des porteurs y sont toujours faibles, inférieures au $\mathrm{cm}^{2} \mathrm{~V}^{-1} \mathrm{~s}^{-1}$, soit en gros au moins 1000 fois plus petites que celle du silicium. Par nature même, il ne faut donc pas s'attendre à pouvoir obtenir des composants rapides avec les milieux organiques.

\section{B. Comment trouver de l'ordre dans le désordre? Conséquences.}

Les milieux organiques sont connus pour être de nature amorphe et présenter une organisation structurale désordonnée. Le schéma de la figure 4 illustre le cas le plus général d'agencement spatial de molécules organiques ; il attire une succession de remarques.

La première est qu'il est possible de trouver des régions de l'espace localement ordonnées parmi l'enchevêtrement des molécules polymères. Il est même possible, ainsi que le montre la figure 5 de trouver des organisations supramoléculaires très sophistiquées. Parmi le fatras de la vue AFM, se

distinguent des poutres d'oligomères constituées de plusieurs fibrilles parallèles entre elles, dans lesquelles les molécules d'oligothiophène de $6,76 \mathrm{~nm}$ de long sont ordonnées perpendiculairement à la longueur de la poutre. Un des objectifs actuels est d'essayer de maîtriser la localisation de ces poutres pour obtenir de la matière ordonnée,

La seconde remarque est qu'en termes de physique du solide il n'existe pas d'ordre à grande distance en trois dimensions dans les matériaux organiques. Très longtemps, il en a été conclu que la méthode de Bloch de recherche des fonctions d'onde électroniques qui exige un milieu périodique de dimension infinie était inapplicable, et que par conséquent de tels milieux ne pouvaient pas présenter de structure de bandes d'énergie électroniques. Il n'y a pas plus bel exemple d'erreur scientifique de raisonnement. La première partie de cette phrase est une affirmation qui va se révéler fausse, quant à la seconde elle constitue simplement un sophisme (l'outil de description n'est peut-être pas le bon).

Montrons qu'il est effectivement possible de trouver un ordre en introduisant une notion nouvelle, celle d'ordre à courte distance. Considérons dans ce but la figure 6 , qui représente le cas d'un oligomère à 15 monomères.

Ainsi, quoique la partie supérieure de la figure 6 soit la représentation d'un milieu désorganisé, il n'en reste pas moins vrai que l'on peut observer le long de l'oligomère $\mathrm{AD}$ à 15 mères, une période à une dimension, dont la longueur est celle du motif répétitif $A B$, différent du monomère $\mathrm{AA}_{1}$; c'est l'ordre à courte distance, par opposition à l'ordre à grande distance des semiconducteurs classiques. Cette notion d'ordre à courte distance ne pourrait qu'être qu'une finesse intellectuelle sans finalité s'il ne s'y greffait pas des considérations énergétiques au niveau des liaisons chimiques. Les forces intramoléculaires représentées par les flèches bleues de la figure 6 sont de types coulombien. Les forces intermoléculaires, si elles sont bien également elles aussi de type coulombien, ont des amplitudes très faibles, ne dépassant pas celles du type Van der Waals, donc très inférieures aux forces intramoléculaires. $\mathrm{Au}$ plan énergétique, on a donc le droit de considérer l'assemblée des oligomères $\mathrm{AD}$ de la figure 6 comme autant de systèmes à une dimension énergétiquement indépendants dans l'espace à trois dimensions.

La conséquence est qu'il est possible d'appliquer à l'oligomère $\mathrm{AD}$ la méthode des fonctions de Bloch, puisque c'est un système périodique à une dimension.

\section{Structure de bandes d'énergie électronique des milieux organiques.}

$\mathrm{Du}$ résultat précédent, si on peut comprendre que l'usage des fonctions de Bloch est possible, il est toutefois nécessaire de tempérer l'enthousiasme qui viserait, pour calculer une structure de bande en milieu organique, à simplement copier ce qui est connu dans les métaux et les semiconducteurs minéraux. Rappelons en effet que la permittivité électrique d'un polymère est toujours supérieure à celle d'un métal. Or on sait que cette permittivité constitue la fonction écran du potentiel local agissant sur les électrons du milieu (20). Il en résulte que la portée du potentiel électrique est beaucoup plus faible dans les milieux organiques que dans les 
métaux ou les semiconducteurs minéraux. Aussi parle-ton pour les électrons d'états localisés en milieu organique par opposition à la notion d'états délocalisés dans les métaux ou les semiconducteurs minéraux. Ces deux notions sont équivalentes en terme de chimie à celles de liaison forte et respectivement de liaison faible. Les fonctions de Bloch, qui décrivent habituellement les orbitales électroniques et qui sont des ondes planes modulées en amplitude et d'étendue infinie, devront subir des modifications pour devenir localisées dans la matière organique, on les appelle fonctions de Wannier. Compte tenu de cette modification, il est alors possible de mettre en œuvre la méthode HFS (Hartree-Fock-Slater) pour calculer la structure de bandes $\mathrm{E}(\mathrm{k})$, $\mathbf{k}$ étant le vecteur d'onde. Le problème, bien évidemment, fait intervenir plus de trois corps puisque, par exemple, un simple oligomère contient plusieurs centaines d'électrons. Sa complexité dépasse les limites rigoureuses d'approximation du physicien. Aussi est-ce grâce à la mise en œuvre de méthodes plus globales, propres au spécialiste de chimie quantique, que le calcul peut aboutir (21). La figure 7 illustre le cas du PMMA (polyméthacrylate de méthyle). Les niveaux profonds occupés de la bande de valence, situés au dessous de -30 $\mathrm{eV}$, ne sont pas représentés, parce qu'en général inintéressants pour l'électronique et l'optoélectronique. Le sommet de la bande de valence occupé par les orbitales $\pi$ est désigné sous le terme de bande HOMO (Highest Occupied Molecular Orbital) ; il est placé dans le PMMA à -9,69 eV, alors que la bande inoccupée la plus basse, appelée LUMO (Lowest Unoccupied Molecular Orbital) est constituée d'une orbitale antiliante $\pi^{*}$ placée à $-4,19 \mathrm{eV}$ (22). La largeur de la bande interdite $\mathrm{E}_{\mathrm{g}}=\mathrm{E}_{\mathrm{LUMO}}-\mathrm{E}_{\mathrm{HOMO}}=5,5 \mathrm{eV}$ montre que le PMMA est un isolant. Par contre, la structure de bande du PPP (poly[para-phénylène]) (Figure 8) fait apparaître une largeur de bande interdite de seulement $2,85 \mathrm{eV}$, indiquant que ce polymère à structure conjuguée, a un comportement de semiconducteur.

\section{La VBDOS.}

La densité des états d'énergie électroniques dans la bande de valence (VBDOS, pour Valence Band Density Of States) est une représentation équivalente à celle de la première zone de Brillouin. Elle présente l'avantage de donner plus directement une indication sur la population électronique en fonction de l'énergie. Mathématiquement, elle se déduit de la représentation $\mathrm{E}(\mathrm{k})$ par une simple opération de dérivation à des coefficients de proportionnalité près :

$$
\mathscr{D}(\mathrm{E})=\mathrm{n} \frac{\pi}{\mathrm{a}} \frac{\partial \mathbf{k}}{\partial \mathrm{E}}(\mathrm{E})=\mathrm{n} \frac{\pi}{\mathrm{a}} \frac{1}{\left.\frac{\partial \mathrm{E}}{\partial \mathbf{k}}(\mathrm{E})\right)}
$$

où a est la longueur du motif répétitif dans la molécule, $\mathrm{n}$ la densité volumique en électrons, et $\mathbf{k}$ le vecteur d'onde. La figure 9 illustre le cas du PMMA et montre l'intérêt de la représentation en VBDOS, puisqu'il est possible d'attribuer une bande à chaque type de liaison chimique.

Revenons maintenant sur le choix de la valeur de $30 \mathrm{eV}$ comme limite inférieure de la représentation de la structure de bandes. Cette limite varie d'un polymère à un autre, mais dans des proportions relativement faibles. Le point remarquable est que la majorité des polymères étudiés montre qu'il existe dans la bande de valence un paquet d'électrons, regroupés entre cette limite et la bande HOMO, séparé du reste des nivaux profonds par quelques dizaines d'eV. Ce domaine d'énergie vide en électrons, sans être une bande interdite, entraîne un comportement particulier des électrons du sommet de la bande de valence. Sans être libres au sens où on l'entend en physique du solide, ces électrons disposent d'une certaine liberté leur permettant d'entrer en oscillations collectives. La bande plasmon résultante est centrée à une fréquence de résonance $\omega_{\mathrm{rp}}$ donnée par :

$$
\omega_{\mathrm{rp}}=\sqrt{\left(\omega_{0 \mathrm{p}}^{2}+\overline{\omega_{\mathrm{i}}^{2}}\right)}
$$

où $\omega_{0 \mathrm{p}}$ est la fréquence plasma pure et $\overline{\omega_{i}^{2}}$ la moyenne quadratique des fréquences électroniques des bandes d'énergie individuelles $E_{\mathrm{i}}=\hbar \omega_{\mathrm{i}}$ (23). Ce résultat a deux conséquences intervenant en électronique nucléaire lors de la détection des rayonnements ionisants, et en technologie de gravure sèche des milieux organiques par faisceaux de particules: l'endommagement du milieu organique est de nature sélective. Les liaisons préférentiellement rompues sont celles dont la bande d'énergie se trouve sous la raie plasmon. En effet, lors du passage de la particule, un transfert résonant d'énergie s'effectue entre les électrons du sommet de la bande de valence et la raie plasmon à la fréquence $\omega_{\text {rp }}$ induite par le champ électrodynamique rayonné par la particule en mouvement. Le tableau 1 donne les principales caractéristiques de la structure de bandes de quelques polymères.

\section{E. Propriétés de transport et dopage des semiconducteurs organiques.}

Les propriétés de transport dans les milieux organiques et notamment les semiconducteurs organiques, essentiellement constitués par les polymères conjugués, peuvent être modélisées en gros à partir des travaux de N. Mott et E. Davis sur les milieux amorphes $(24,25)$. Le dopage des polymères conjugués introduit les notions de polaron et de bipolaron, désignation des quasi-particules associées à l'introduction dans la chaîne organique d'une charge simple et de la déformation élastique résultante dans la chaîne, et respectivement d'une double charge et de la déformation associée $(26,27)$. Sans entrer dans les détails, et pour faire simple, pour un polymère conjugué, dont on notera de manière générique par Pol la molécule,

Le dopage de type $\mathrm{n}$ conduit à l'état $\mathrm{Pol}^{-}$, correspondant à l'introduction d'un, voire plusieurs 
électrons par monomère, c'est-à-dire en terme de chimie à la forme réduite de la molécule. Les principaux éléments dopants sont en conséquence les alcalins, $\mathrm{Li}, \mathrm{Na}, \mathrm{K}$, et Cs.

Le dopage de type $\mathrm{p}$ conduit à l'état $\mathrm{Pol}^{+}$, correspondant à la perte d'un électron, voire plusieurs, par monomère, soit à la forme oxydée de la molécule. Aussi, dans ce cas, les candidats au dopage sont les halogènes, $\mathrm{F}, \mathrm{Cl}, \mathrm{Br}$ et $\mathrm{I}$.

La forme dopée $\mathrm{n}$ est difficile à maintenir en l'état à l'air libre, en raison de la diffusion naturelle de l'oxygène qui effectue immédiatement un contre-dopage. La présence d'une couche de protection en surface est nécessaire pour effectuer un blocage à cette diffusion (packaging). Ce problème est général à tous les composants organiques pour cette raison. Une autre façon de procéder est d'effectuer un maillage serré du polymère en surface lors de sa synthèse (13).

A propos des différents types de conduction, rappelons simplement les principaux modèles de conduction continue rencontrés dans les matériaux conjugués; en effet, les différentes variantes de conduction en alternatif ne se distinguent pas suffisamment entre elles pour pouvoir identifier de façon certaine un type de transport. La figure 11 localise dans la structure de bandes les états électroniques mis en cause par chacun des types de conduction, alors que la figure 12 les classe suivant l'activation thermique. Schématiquement, on retiendra quatre types de conduction continue $(26,27)$ :

(I) : les états délocalisés en dehors du gap, qui s'apparentent au mode de conduction "drift" rencontré dans les semiconducteurs classiques; c'est le cas des polyacéthylènes.

(II) : les états localisés dans les pieds de bande. Il s'agit d'une conduction où la mobilité est thermiquement activée. On la rencontre dans les polymères conjugués. S'y superpose dans ce modèle la bande polaronique pour les polymères dopés.

(III) et (IV): les états localisés autour du niveau de Fermi, liés à l'existence de bandes de défauts du type bandes de Hubbard. Le mode de transport est celui par saut dans les deux cas, assisté thermiquement et par effet tunnel. Pour (III) l'assistance thermique est faible correspondant à un saut au plus proche voisin, alors que dans le cas (IV), l'assistance thermique est plus forte pour des sauts à distance variable. Ce dernier cas est sensiblement celui du mode de conduction dans les polymères initialement isolants et rendus conducteurs par production de défauts (28).

Expérimentalement, le dopage peut être effectué par diffusion thermique ou par implantation ionique. Etant donné que par diffusion thermique il est impératif de rester sous la température de transition vitreuse, il n'est pas possible d'effectuer de la diffusion en sites profonds. Le dopage résultant est instable au cours du temps; au bout de quinze jours à peine la moitié de la concentration en espèces dopantes est restée piégée au sein du matériau. Par opposition, le dopage par implantation ionique produit un dopage stable sur plus de dix ans (26). Etant donné qu'un recuit thermique est inopérant après une simple implantation pour uniformiser la concentration en éléments dopeurs dans la couche implantée, il est nécessaire dans ce but de faire appel à des variantes comme l'IBAD (Ion Beam Assisted Deposition) (29).

\section{QUELQUES COMPOSANTS ELEMENTAIRES}

A travers une étude particulière du fonctionnement et des conditions de fabrication de deux composants que nous avons choisis parce que typiques de l'optoélectronique organique, la diode électroluminescente et le cristal photonique, nous allons toucher de plus près les aspects spécifiques de la voie organique, avantages comme inconvénients, pour finalement envisager les directions dans lesquelles les efforts se poursuivent ou sont à entreprendre dans l'orientation d'une production à bas coût.

\section{A. Les diodes électroluminescentes organiques.}

Les propriétés électroluminescentes de l'anthracène sont connues depuis longtemps. Cependant la taille de ses cristaux entraîne une épaisseur trop élevée des couches formées dans une structure de diode. Les tensions nécessaires à la stimulation de l'électroluminescence sont de l'ordre de la centaine de volts, incompatible avec un usage courant (30). Les études entreprises pour diminuer cette tension ont constitué un des enjeux des années 70 et 80. Elles ont finalement débouché sur deux catégories de matériaux capables de manifester une électroluminescence suffisante en couche mince. La première est constituée de ce qu'il est convenu d'appeler "les petites molécules", qui sont formées de complexes du type $\mathrm{Mq}_{3}$, où $\mathrm{M}$ est un métal de la colonne IIIA ( $\mathrm{Al}$, $\mathrm{Ga}$, In) et q le ligand 8-tris-hydroxyquinoline ; l'initiateur en est C. Tang (31), et il est habituel de désigner par OLED (Organic LED) la diode résultante. Certains polymères $\pi$ conjugués, telles que le PPV (poly[phénilène-vinylène]) et ses dérivés, ou le PPP, le PTh (poly[tiophène]), le PVK (poly[vinyl-carbazole]) constituent la seconde catégorie. L'étude de leur interface avec Li, Ca, Mg, Al par J. Burroughes (32) a débouché sur la seconde forme de diodes électroluminescentes, les PLEDs (pour Polymer LEDs). Chacun de ces deux types de matériaux ont leurs avantages et leurs inconvénients. Au départ, on a cru que l'efficacité quantique de luminescence était plus élevée dans les petites molécules, parce que les propriétés de transport des électrons y sont moins bonnes que le long de la chaîne d'un polymère. A la base du raisonnement était la plus mauvaise mobilité des électrons et des trous dans les petites molécules ; celle-ci favoriserait la durée de vie des paires électron-trou (exciton) facilitant à priori la probabilité de recombinaison radiative. On verra plus 
loin qu'en fait il n'en est rien. Tout dépend principalement de la structure de la diode choisie, et des épaisseurs des couches notamment. Que ce soit les OLEDs ou les PLEDs, toutes ces diodes organiques offrent des performances supérieures aux diodes inorganiques en résolution, contraste, luminosité, qualité et choix des couleurs, rapidité de réponse, ouverture angulaire, puissance dissipée, poids, et même coût, à condition d'être associées pour leur commande à des transistors couche mince $\mathrm{p}-\mathrm{Si}$ (p-Si TFT). Il n'est que leur durée de vie, qui dépasse juste $10000 \mathrm{~h}$, pour laquelle les dispositifs p-Si TFT LCD ou a-Si TFT LDC font mieux. La raison tient dans le problème de la diffusion de l'oxygène de l'air, cité précédemment. Le remède est le même que celui rencontré il y a trente ans dans les composants GaAs, l'encapsulation.

La structure d'une diode luminescente est représentée sur le schéma de la figure 13. le matériau électroluminescent, classiquement d'épaisseur de l'ordre de $100 \mathrm{~nm}$, est pris en sandwich entre cathode et anode. Cette dernière est déposée sur un substrat transparent, feuille organique ou verre pour mieux éviter la contamination par l'oxygène. Elle doit être elle-même transparente; c'est pourquoi devant en même temps permettre l'injection de trous, donc bien conduire, seul l'oxyde mixte indium/zinc (ITO) réalise le compromis. Si sa réalisation sur verre est classique, celle sur substrat organique avec de bonnes performances, aussi bien en transmission optique qu'en conduction électrique, est un des enjeux actuels de l'optoélectronique organique de façon générale. La figure 15 illustre les résultats obtenus au laboratoire par B. Lucas et W. Rammal sur CR39 dans le cadre d'applications non seulement pour les LEDs, mais aussi en vue du transistor transparent. Des valeurs similaires ont été atteintes sur polyéthylène (PET). Le mécanisme de fonctionnement d'une LED est illustré sur la figure 14. Il peut être résumé ainsi : les électrons sont injectés à la cathode pour former un polaron $\mathbf{P}^{-}$, alors que les trous sont injectés à l'anode dans le matériau électroluminescent pour constituer le polaron $\mathbf{P}^{+}$. Les deux polarons de signes opposés migrent sous l'effet du champ électrique à l'encontre l'un de l'autre pour se recombiner sous la forme d'un exciton qui possède quatre état possibles de spin selon le schéma de la figure 16. Il est habituellement admis que l'extinction de l'exciton ne s'effectue de façon radiative qu'à partir de l'état singulet. Par molécule, le taux de recombinaison radiative $\mathrm{r}_{\mathrm{st}}$ ne peut donc pas dépasser $25 \%$. Le rendement efficace d'électroluminescence $\eta_{\text {eff }}$ est donné par le produit :

$$
\eta_{\text {eff }}=\gamma \tau_{\mathrm{r}} \mathrm{r}_{\mathrm{st}} \eta_{\mathrm{ext}}
$$

où :

$\gamma$ est le taux de recombinaison des porteurs injectés, donc en fait le nombre d'excitons produits sur le nombre de paires électron-trou injectées,

$\tau_{\mathrm{r}}$ représente l'efficacité de transition radiative de l'exciton singulet, $\mathrm{r}_{\mathrm{st}}$ est le taux réel des excitons donnant lieu à une recombinaison radiative, donc des états excitoniques singulets,

$\eta_{\text {ext }}$ est le couplage optique externe, faisant intervenir les pertes de lumière sur la face de sortie.

En général $\tau_{\mathrm{r}}$ est proche de 1 , mais il arrive que près des interfaces où apparaissent des états parasites, nés de l'existence de centres de défauts qualifiés de tueurs, l'exciton soit annihilé sans donner lieu à une transition radiative. De plus, au niveau de la cathode se manifeste une absorption de ces excitons par résonance plasmon, limitant encore l'efficacité de luminescence. Avec les petites molécules, où n'existent pratiquement pas d'interactions intermoléculaires, $r_{\text {st }}$ ne dépasse pas $25 \%$. $\mathrm{Au}$ contraire avec les polymères, et contrairement au raisonnement ci-dessus, le processus de capture électrontrou est favorisé par la délocalisation des orbitales le long de la chaîne, induisant une interaction à grande distance $(10 \mathrm{~nm})$ qui permet une production en plus grande proportion des états singulets, puisqu'on n'est pas restreint à considérer les états de spin sur une molécule, soit un mère (33). Ainsi, CDT atteint jusqu'à 50\% d'efficacité de transition radiative avec les dérivés du PPV (34).

Abordons maintenant le phénomène de transport des charges amenant les états polaroniques à leur recombinaison en états excitoniques dans le milieu électroluminescent. Les figures 17 et 18 schématisent le processus lorsque tout se passe comme prévu, c'est-à-dire avec :

un travail de sortie de la cathode plus petit que le niveau de la LUMO du matériau luminescent,

un travail de sortie de l'anode au dessus de la bande HOMO,

la rencontre des deux états polaroniques $\mathrm{P}^{-}$et $\mathrm{P}^{+}$au sein du milieu électroluminescent.

Dans ce type de configuration élémentaire, plusieurs améliorations ont été apportées successivement afin d'augmenter l'efficacité de recombinaison. Les trous étant plus lents que les électrons, une quantité non négligeable d'électrons atteint l'anode sans avoir rencontré de trous. Il faut faciliter l'injection des trous à l'anode et leur transport. Dans ce but est inséré antre l'anode et le milieu électroluminescent un matériau transporteur de trous et en même temps bien adapté au plan du travail de sortie entre celui de l'anode et celui du milieu électroluminescent. On ajoute en plus souvent une couche de bocage d'électrons entre cette couche et le milieu électroluminescent. Les matériaux les plus connus réalisant cette fonction sont le TPD, le mTADATA, le $\alpha$ NPD, le HTM-1 ou le TPTE. Si le milieu électroluminescent n'est pas un bon transporteur d'électrons, on le ramène à une couche très fine et on insère entre lui et la cathode un bon transporteur d'électrons possédant un travail de sortie compris entre la LUMO et le potentiel de surface de la cathode. Bien d'autres configurations sont possibles, dont par exemple celle représentée figure 19 . 
La figure 20 représente dans sa généralité les formes d'évolution des caractéristiques courant - tension I(V), et Luminance - tension L(V). La nature de la conduction et les conditions d'injection aux interfaces métal/isolant sont un sujet connu depuis la fin des années 70 (35); il a fait l'objet d'adaptation plus fine au cas des molécules concernées dans les OLEDs ou les PLEDs (36). Disons simplement en résumé que les formes des courants diffèrent suivant que les contacts sont ohmiques ou pas. Il est généralement admis que le contact peut être considéré comme ohmique si la barrière de potentiel métal/polymère $\varphi_{\mathrm{MP}}$ est inférieure à $0,6 \mathrm{eV}$. Dans ce cas, c'est le volume du polymère qui détermine la nature de la conduction, et plusieurs situations peuvent se présenter :

la loi ohmique

la loi SCL (courant limité par la charge d'espace)

la loi TCL (courant limité par les pièges)

Pour des contacts non ohmiques, ce sont les conditions d'injection aux électrodes qui imposent la nature du courant. On rencontre alors les cas d'émission

thermoélectronique,ou

Schottky,ou

Fowler-Nordheim (effet tunnel).

Lorsque, cas classique, le choix des matériaux est convenablement fait par rapport aux barrières $\varphi_{\text {MP }}$ pour les deux électrodes, on se trouve dans le cas d'un contact ohmique. C'est la charge d'espace aux électrodes qui constitue alors le goulot d'étranglement; on se situe dans le cas du modèle SCL. Par exemple la densité en courant d'électrons de la structure de la figure 19 est donnée par la loi :

$$
J=\frac{9}{8} \mu_{0} \varepsilon_{0} \varepsilon_{r} \frac{V^{2}}{L^{3}} e^{0,89 y \sqrt{E}}
$$

où la mobilité $\mu$ suit une loi du type $\mu_{0} \mathrm{e}^{0,89 \gamma \sqrt{\mathrm{E}}}\left(\mu_{0}\right.$ représentant la mobilité en champ nul), E étant le champ appliqué, L l'épaisseur du polymère électroluminescent et $\varepsilon_{\mathrm{r}}$ sa permittivité relative.

Les évolutions des structures LEDs organiques et polymères se font par rapport à :

l'accroissement de leur durée de vie, et débouchent dans ce cas sur l'encapsulation. Des procédés physiques nouveaux de polymérisation in situ (VDP: Vapour Deposition Polymerisation) présentent de ce point de vue un grand intérêt,

la nature des substrats afin de produire des dispositifs nomades, donc vers la flexibilité, mais aussi pour rendre le produit consommable. Ainsi, peut-on envisager des LEDs organiques sur du papier journal, en utilisant des matériaux de planarisation (ORMACER) (37),

le contrôle direct des barrières de potentiel par le moyen des "soft lithographies" où la technique de report des couches métalliques par tampon en PDMS (Poly(DiMéthyle-Siloxane]) permet de diminuer les $\varphi_{\text {MP }}$ par rapport au procédé classique d'évaporation sous vide
(38). L'ensemble de ces voies douces présente de façon générale l'avantage de se soustraire à l'effet de diffusion du métal à travers la couche organique, autorisant l'emploi de couches fines très, inférieures à 100 nm, sans risque de court-circuit.

des techniques de dépôt rapide, comme l'impression par jet d'encre (Société Epson).

\section{B. Les dispositifs à bande interdite organique (BIPO).}

Malgré de nombreuses tentatives, il n'existe pas à notre connaissance de réalisation de BIPO au moyen de procédés de type "bottom up". Dans le cadre des procédés de type "top down", afin d'abaisser le coût de fabrication, il est adroit de les envisager suivant la voie des lithographies douces. Quelle que soit la méthode envisagée, procédé de réplication par moulage, emboutissage, etc..., le tampon mère est à fabriquer dans des géométries de dimensions sub-microniques, ou juste microniques. La détermination des dimensions autorisant l'ouverture d'une bande interdite n'est pas banale. Elle nécessite l'emploi de méthodes numériques relativement lourdes. Ensuite, la fabrication du tampon à ces échelles, relativement courantes en microélectronique classique, implique en technologies "tout organique" des difficultés d'ordre purement technologiques. Les méthodes de lithographie classiques en phase humide sont à déconseiller, en raison de la très grande sensibilité des organiques aux solvants polaires, comme l'acétone. L'usage de la voie sèche est fortement recommandé. Nous allons envisager successivement les deux étapes, celle du calcul des géométries, puis celle de la fabrication de la structure.

La première condition à ne pas perdre de vue est la condition de guidage dans le milieu organique (permittivité électrique $\varepsilon_{\mathrm{g}}$ ), placé entre le substrat $\left(\varepsilon_{\mathrm{s}}\right)$ et le surstrat $\left(\varepsilon_{\mathrm{a}}\right)$, en général l'air : $\varepsilon_{\mathrm{g}}>\varepsilon_{\mathrm{s}}>\varepsilon_{\mathrm{a}}$. L'ouverture d'une bande interdite photonique résulte d'une rétrodiffusion multiple cohérente localisant les fonctions d'ondes associées aux photons à l'intérieur de la matière. On se retrouve dans les mêmes conditions que celles d'une bande interdite électronique; le phénomène se produit pour chaque longueur d'onde vérifiant dans l'espace la condition : $\lambda=n \pi / a$, où $n$ est un entier et $a$ la période du milieu (condition de Brillouin pour le plan de rang $n$ ). Les milieux organiques étant en général désorganisés, il est nécessaire de fabriquer la structure périodique. Le programme de calcul devra donc satisfaire à deux conditions antagonistes : interdire la propagation de certains modes, et au contraire autoriser celle d'autres. La mise en œuvre de la méthode des ondes planes (PWM, Plan Wave Method) travaillant dans l'espace des vecteurs d'onde $\boldsymbol{k}$ par la technique des différences finies dans le domaine temporel (FDTD, Finite-difference Time-Domain) permet de déterminer les modes dont la propagation est autorisée (39, 40). Résumons les principaux résultats $(41,42)$. Pour une bande interdite située autour de $1,55 \mu \mathrm{m}$, la valeur de $a$ est de $0,775 \mu \mathrm{m}$ 
pour une épaisseur des motifs organiques diffractifs de 1 $\mu \mathrm{m}$ et une épaisseur de substrat supérieure à 1,55 $\mu \mathrm{m}$. La largeur de la bande interdite photonique $\Delta \lambda$, placée entre le mode $L A M U B$ (Lowest Allowed Mode in the Upper Band) et le mode $H A M I B$ Highest Mode in the Infra Band) est obtenue approximativement en divisant par deux la constante du réseau $a$. Les calculs montrent cependant qu'elle dépend très fortement des conditions de polarisation et de la classe cristalline du réseau. La polarisation TM est plus favorable à l'ouverture du gap que la polarisation TE. Un réseau cristallin triangulaire, pour des dimensions identiques, ouvre toujours une bande interdite plus large qu'un réseau carré. Il se peut même qu'existe un gap significatif en mode TM dans un réseau triangulaire sans qu'il en apparaisse un pour le réseau carré de caractéristiques géométriques identiques. La figure 21 effectue la comparaison entre les deux types de géométries pour une polarisation TM. Un résultat important concerne ce qu'il est convenu d'appeler improprement le contraste d'indice, $\varepsilon_{\mathrm{g}} / \varepsilon_{\mathrm{s}}$. La figure 22 montre l'évolution de la largeur de la bande interdite en mode TM et réseau triangulaire autour de $\lambda=1,55 \mu \mathrm{m}$ en fonction de $\varepsilon_{\mathrm{g}} / \varepsilon_{\mathrm{s}}$. La bande interdite n'apparaît que pour des contrastes supérieurs à 2,13, correspondant à un rapport d'indice optique $\mathrm{n}_{\mathrm{g}} / \mathrm{n}_{\mathrm{s}}=1,45$. Si l'on envisage un BIPO tout organique, cette condition ne peut pas être remplie actuellement. En effet, parmi les polymères transparents, les limites inférieures et supérieures en indice optique sont respectivement 1,42 et 1,74 , impliquant une valeur maximale de $n_{g} / n_{s}=1,22$. Dans ce secteur, des progrès sont attendus par rapport aux deux points suivants :

dans le domaine de la chimie, la réalisation de polymères de très haute densité (haut indice) et respectivement de basse densité,

dans le domaine de la mesure, la détermination précise des permittivités électriques des matériaux organiques vers $1,3 \mu \mathrm{m}$ et $1,5 \mu \mathrm{m}$.

Nous avons entrepris la fabrication du tampon par un procédé sec entièrement physique, la gravure non réactive par faisceau d'ions focalisé (FIB, Focused Ion Beam). La machine a été construite pour être entièrement dédiée à la matière organique, en raison d'un dégazage important en cours de gravure, et afin d'effectuer une imagerie par microscopie ionique à balayage, avec un détecteur spécialement adapté aux énergies des électrons secondaires générés au cours de l'interaction ion matière organique. Rappelons de ce point de vue qu'une imagerie classique au microscope électronique à balayage détruit la structure organique submicrométrique. Les capacités théoriques de focalisation de la machine sont comprises entre $10 \mathrm{~nm}$ et $30 \mathrm{~nm}$. La figure 23 montre deux échantillons de BIPO obtenus dans le CR39 sur support d'aluminium. La difficulté consiste dans la matière organique à réduire les effets de diffusion thermique responsables d'un élargissement de l'impact du spot beaucoup plus critique que dans les minéraux. C'est la raison pour laquelle il est important pour cumuler la fluence en ions nécessaire à la gravure de procéder par une série d'impacts à l'endroit à graver, séparés entre eux par un temps de rafraîchissement permettant au polymère de ne pas dépasser localement sa température de transition vitreuse, ou de décomposition, en gros empêcher le fluage. L'usage d'un outil entièrement numérique, pour programmer et piloter le faisceau d'ions, s'impose.

Les matériaux organiques qui se prêtent le mieux à la gravure sont ceux qui produisent en cours de gravure sous le faisceau d'ions des espèces secondaires de masse moléculaire élevée. Des masses de 135 u.ma. peuvent être atteintes avec le CR39, alors qu'avec le PMMA la limite est 107 u.m.a., rendant ce polymère moins facile à graver vers les petites dimensions.

\section{IV- CONCLUSION}

Les milieux organiques offrent tout un panel de dispositifs qui peuvent dès maintenant être mis dans les circuits commerciaux, notamment les dispositifs d'affichage. Il n'est pas impossible que les études menées dans le sens d'une production bas coût débouchent à moyen terme vers des produits consommables.

L'horizon d'un réseau local de communications "tout organique" n'est plus une utopie, quand bien même des progrès soient encore nécessaires, comme l'ont montré les lignes précédentes.

Par ailleurs, des niches d'applications non abordées ici sont offertes toutes grandes dans beaucoup de domaines, notamment le secteur des capteurs de la biochimie et de la biologie, pour lesquels les matériaux organiques sont tout naturellement biocompatibles. Associés à un réseau de communications, ils sont tout désignés pour la surveillance médicale à distance.

\section{REFERENCES}

[1] J. Burroughes (communication privée)

[2] Materialstoday, Juillet - août 2005

[3] X. Liu, D. Poitras, Y. Tao, C. Py, Can. Phys. 82(6), 481, 2004

[4] J. Zyss, http://www.lpqm.enscachan.fr/recherche\%20microcavites

[5] C. Cornic, B. Lucas, A. Moliton, Polymer Interenational, 53, 1979, 2004.

[6] Sinyukov, M. Hayden, J. Phys. Chem., B 108, 8515, 2004

[7] D.G. Girton, S.L. Kwiatkovski, G.F. Lipscomb, R.S. Lytel, Appl. Phys. Lett., 58, 1730, 1991

[8] C. Cornic, B. Lucas, A. Moliton, B. Colombeau, P. Raynaud, EMRS'01 Spring Meeting, 5-8 juin 2001, Strasbourg

[9] C. Cornic, B. Lucas, A. Moliton, B. Colombeau, R. Mercier, Synthetic Metals, 127, 299, 2002

[10]D. Chiron, T. Trigaud, J.P. Moliton, Synthetic Metals, 124, 33, 2001

[11]B. Lucas, B. Ratier, A. Moliton, J.P. Moliton, T.F. Otero, C. Santamaria, E. Angulo, J. Rodriguez, Synthetic Metals, 55 /57, 1459, 1993. 
[12] J. Rodriguez, J.P. Moliton, T. Trigaud, T.F. Otero, H. Grande, Materials Research Society Symposium Proceedings, 413, 595, 1996.

[13] J. Rodriguez, H. Grande, T.F. Otero, T. Trigaud, J.P. Moliton, Synthetic Metals, 83, 201, 1996.

[14] T. Trigaud, J.P. Moliton, C. Jussiaux, B. Mazière, Nuc. Instrum. Methods, B 107, 323, 1996.

[15] T. Trigaud, J.P. Moliton, C. Jussiaux, B. Mazière, Nuc. Instrum. Methods, B 111, 254, 1996.

[16]Matériaux polymères, Enjeux et perspectives, ouvrage collectif de «L'observatoire français des techniques avancées », Collection ARAGO, tome 16, Masson, Paris 1995.

[17]R.S. Kohlman, A. Epstein in Handbook of Conducting Polymers, seconde edition, Ed.: T.A. Skotheim, R.L. Elsenbauer, J.R. Reynolds; chap. 3:"Insulator-Metal Transition and Inhomogeneous Metallic State in Conducting Polymers", Marcel Dekker,Inc., New York, 1998

[18]H. Shirakawa, E.J. Louis, A.G. Mac Diarmid, C. Chiang, A.J. Heeger, J. Shem. Soc. Chem. Commun., 578, 1977.

[19]C.K. Chiang, M.A. Druy, S.C. Gua, A.J. Heeger, A.G. Mac Diamid, Y.W. Park, J. Am. Chem. Soc.100, 1013, 1978 .

[20]J.M. Ziman, Chap. 5, Electron-electron interaction, in Principles of the theory of solids, seconde édition, Cambridge Univ. Press, 1972.

[21] J.L. Brédas, J. Cornil, F. Meyers, D. Beljone, Electronic structure and optical response of highly conducting and semiconducting conjugated polymers and oligomers, Chap.1 in "Handbook of conducting polymers", seconde edition, New York, 1998.

[22] J.P. Moliton, C. Jussiaux, T. Trigaud, Phil. Mag. B 73, $\mathrm{n}^{\circ} 5,763,1996$.

[23] J.P. Moliton, C. Jussiaux-Devilder, T. Trigaud, Phil. Mag. B 79, n5, 793, 1999.

[24]N.F. Mott, E.A. Davis, Electronic processes in noncrystalline materials, Clarendon Press, 1971.

[25] N.F. Mott, Conduction in non-crystalline materials, Oxford Science Publication, 1993.

[26] Moliton, C. Moreau, J.P. Moliton, G. Froyer, Nucl. Instr. Methods, B 80/81,1028, 1993.

[27] Moliton, Propriétés de transport électronique : IConduction dans les états délocalisés, localisés, polaroniques, Chap. 5 in "Optoélectronique moléculaire et polymère", Collection scientifique et technique des télécommunications, Springer, Paris, 2003.

[28] J.P. Moliton, T. Trigaud, B. Mazière, C. Aubry, Materials Science in Semiconductor Processing, 3, 419, 2000.

[29]R. Anthony, A. Moliton, D. Troadec, Synthetic Metals, 121, 1749, 2001

[30]M. Pope, C.E. Swenberg, Electronic processrs in organic crystals, Clarendon Press, 1982.

[31]C.W. Tang, S.A. Van Slyke, Appl. Phys. Lett. 61, 913, 1987.

[32]J.H. Burroughes, D.D.C. Brabley, A.R. Browon, R.N. Marks, K.D. Mackkay, R.H. Friend, P.L. Burn, A.B. Holmes, Nature, 347, 539, 1990.

[33] J. Beljone, etal., J. Chem. Phys., 102, 2042, 1995.

[34] J. Wilson, A. Köhler, Nature, 413, 828, 2001.

[35]P. Hesto, The nature of electronic conduction in thin insulating layers, chap. 5 in "Instabilities in Silicon Devices", vol.1, Ed.: G. Barbotin, A. Vapaille; North Holland, Amsterdam, 1986.
[36]Moliton, Mécanismes d'injection et de transport dans les milieux résistifs, Chap. 6 in "Optoélectronique moléculaire et polymère", Collection scientifique et technique des télécommunications, Springer, Paris, 2003.

[37]R. Lamprecht, cité p. 418 dans MRS Bulletin, 30, $n^{\circ} 6$, juin 2005

[38] T.W. Lee, J.W.P. Hsu, J. Nanoeng.Nanosyst. soumis.

[39] Tofle, Computational Electrodynamics - The finitedifference-time-domain Method, Ed.:Artech House, New-York, 1995.

[40]S.G Johnson, S. Fan, P.R. Villeneuve, J.D. Joannopoulos, L.A. Kolodziejski, Phys. Rev. B, 60 $\mathrm{n}^{\circ} 8,5751,1999$.

[41]E. Pialat, T. Trigaud, V. Bernical, J.P. Moliton, EMRS'04, Symposium G: Current trends in nanoscience-from mtyerials to applications, $n^{\circ}$ G/PIII04, Strasbourg, 24-28 mai 2004.

[42]E. Pialat, T. Trigaud, V. Bernical, J.P. Moliton, Materials Science and Engineering C, 2005, sous presse. 
TABLEAU 1 VALEURS DES POSITIONS DES BANDES HOMO ET LUMO, DE LA LARGEUR DE BANDE INTERDITE E $_{\mathrm{G}}$, DE LA FREQUENCE DE RESONANCE PLASMON $\omega_{\mathrm{RP}}$, ET DE LA PERMITTIVITE ELECTRIQUE RELATIVE THEORIQUE $\varepsilon_{\mathrm{R}}$ D'UN MATERIAU SANS DEFAUT, POUR QUELQUES POLYMERES.

\begin{tabular}{|c|c|c|c|c|c|}
\hline Polymère & $\mathrm{E}_{\mathrm{HOMO}}(\mathrm{eV})$ & $\mathrm{E}_{\text {LUMO }}(\mathrm{eV})$ & $\mathbf{E}_{\mathbf{g}}(\mathrm{eV})$ & $\omega_{\mathrm{rp}}(\mathrm{eV})$ & $\begin{array}{c}\varepsilon_{r}(0)= \\
1+\left(\hbar \omega_{r p} / E_{g}\right)^{2}\end{array}$ \\
\hline Poly(Ac)trans & -4.7 & -3.3 & 1.4 & 22 & 248 \\
\hline Poly(Ac)cis & -5.1 & -3 & 2.1 & & \\
\hline PPP & -7.5 & -4.7 & 2.8 & 21.3 & 40 \\
\hline PPy & -3.9 & -0.3 & 3.6 & & \\
\hline PPV & -5.2 & -2.7 & 2.5 & & \\
\hline CN-PPV & -5.55 & -2.7 & 3.02 & & \\
\hline MEH-CN-PPV & -5.55 & -3.1 & 2.45 & & \\
\hline PVK & -5.8 & -2.7 & 3.1 & & \\
\hline PMMA & -9.69 & -4.19 & 5.5 & 21.5 & 16 \\
\hline TMA-ODA & -8.14 & -4.90 & 3.25 & 22.3 & 48 \\
\hline PMDA-ODA & -7.9 & -4 & 3.9 & & \\
\hline PC & -8.7 & -2.83 & 5.87 & 21.6 & 14.5 \\
\hline CTA & -10.8 & -4.27 & 6.53 & 22.00 & 12.3 \\
\hline CDN & -10.91 & -5.03 & 5.88 & 22.5 & 15.6 \\
\hline Cellulose & -10.88 & -0.318 & 10.56 & & \\
\hline & & & & & \\
\hline
\end{tabular}




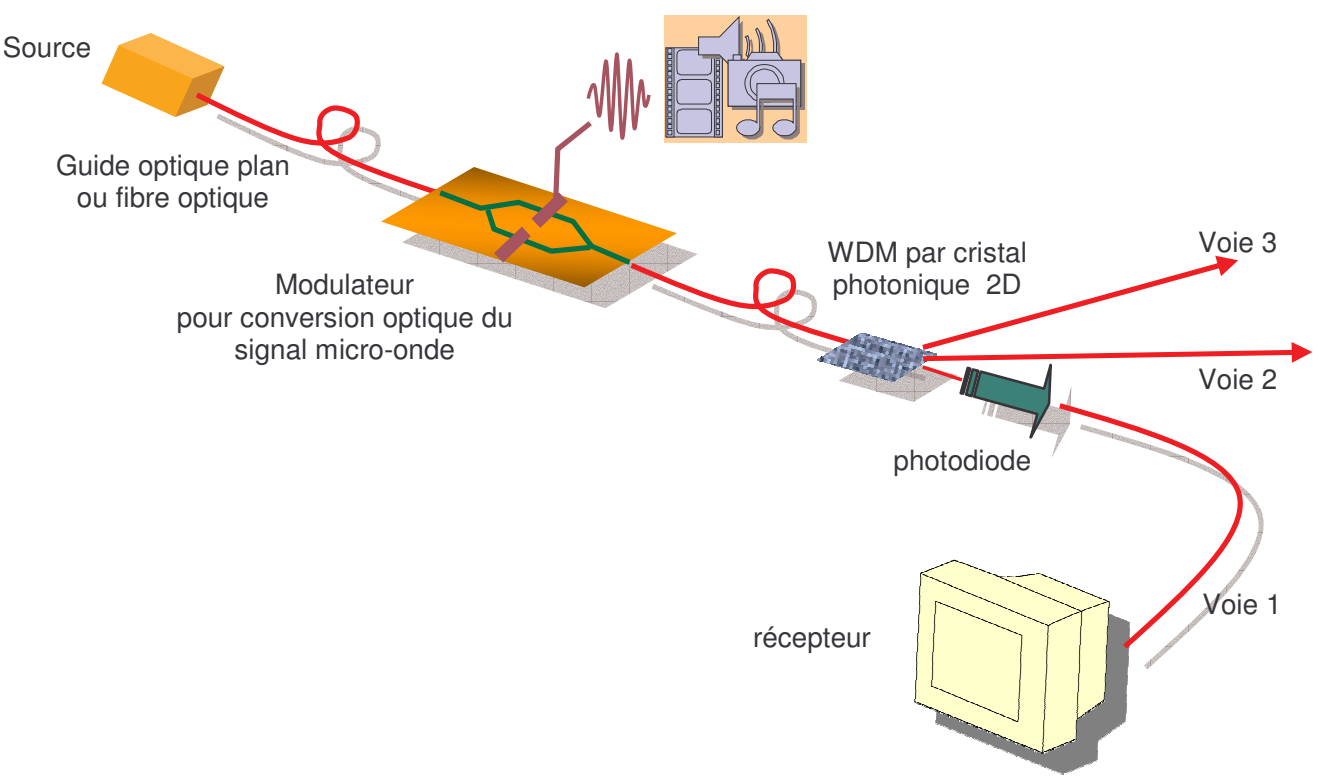

Fig. 1 Schéma d'un réseau de communications rassemblant les dispositifs essentiels, qui sont tous fabricables par des procédés à base de matière organique.

(a)

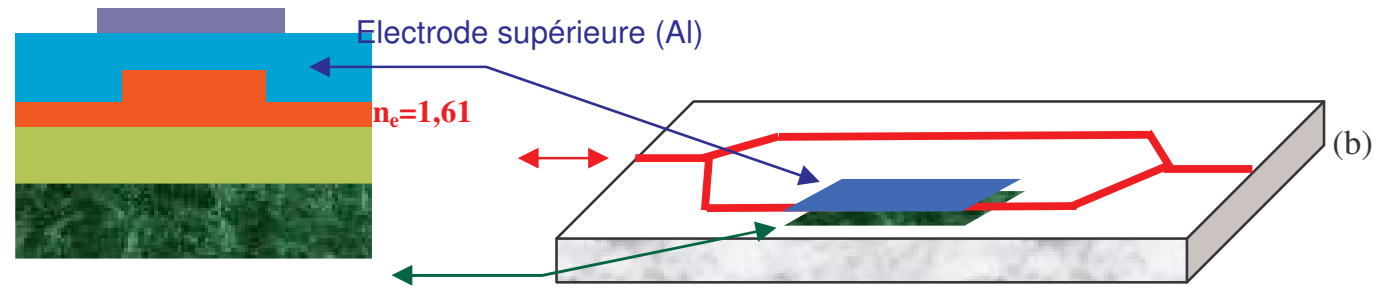

Electrode inférieure $(\mathrm{Si}<100>$ dopé)

Fig. 2 Schéma en coupe de la zone active par effet électro-optique (a) d'un modulateur type Mach-Zehnder (b). Le polymère non linéaire est représenté en rouge ; il est entouré d'une couche tampon supérieure (bleu cyan) et inférieure (vert clair) dont les indices sont légèrement inférieurs. On s'arrange pour que les géométries produisent dans la zone active un champ électrique aussi homogène que possible, et soient compatibles avec les conditions de guidage. 


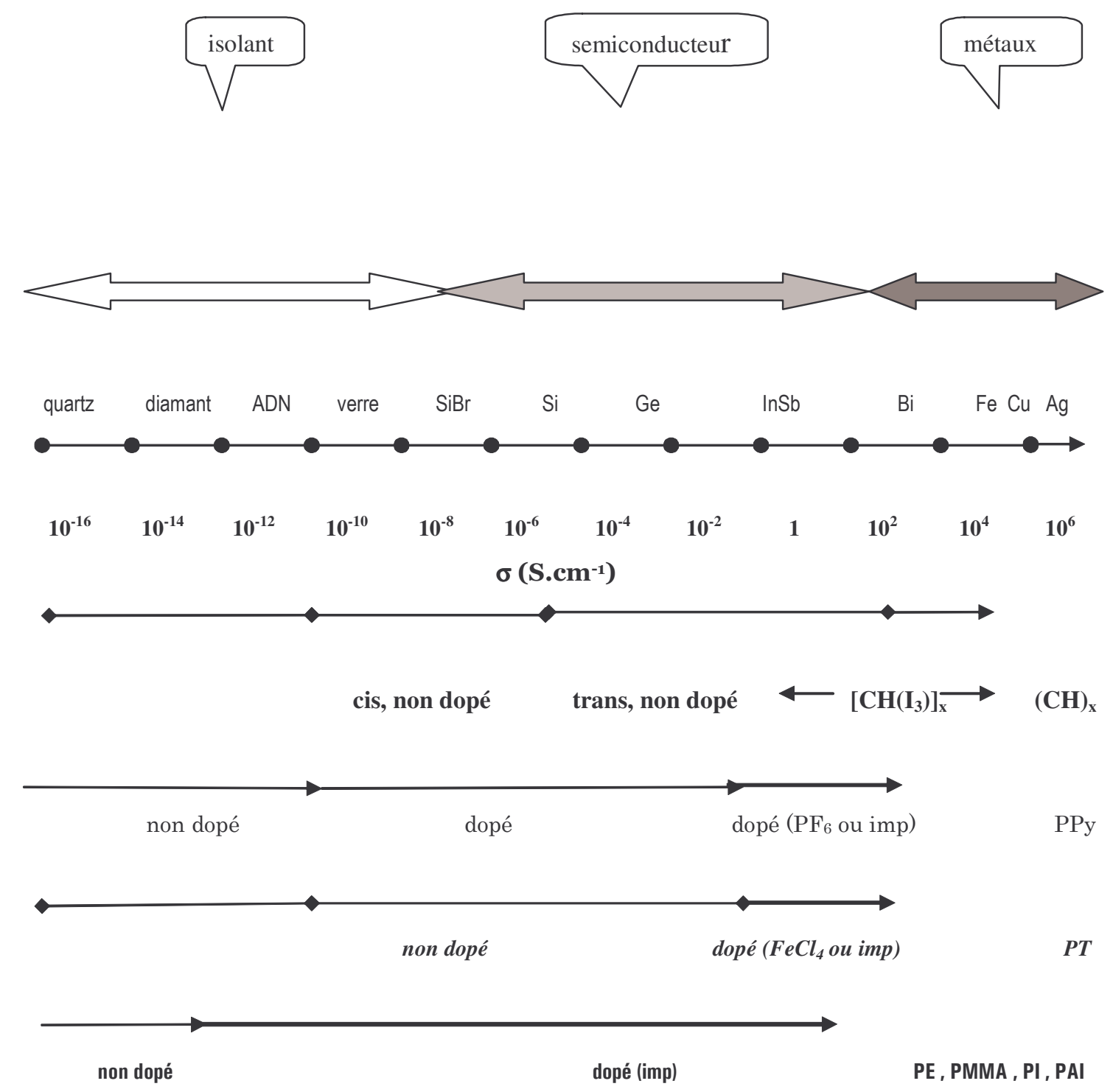

Fig. 3 Echelle et domaines de variation de la conductivité du polyacétylène $(\mathrm{CH})_{\mathrm{x}}$, du polypyrrole $(\mathrm{PPy})$, du polythiophène $(\mathrm{PT})$, du polyétylène (PE), du poly(métacrylate de méthyle) (PMMA), des polyimides (PI), et poly(amido-imides) (PAI); imp :implantation ionique.

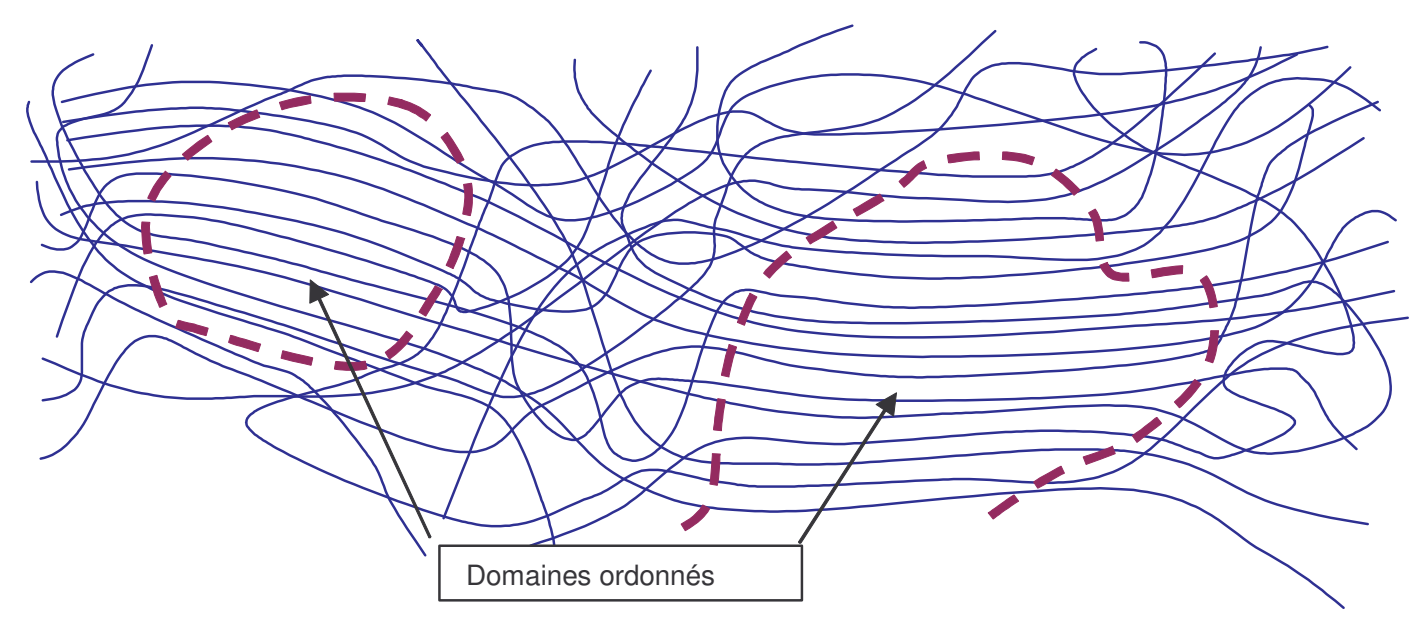

Fig. 4 Domaines de l'espace spatialement ordonnés dans un milieu globalement désordonné. 


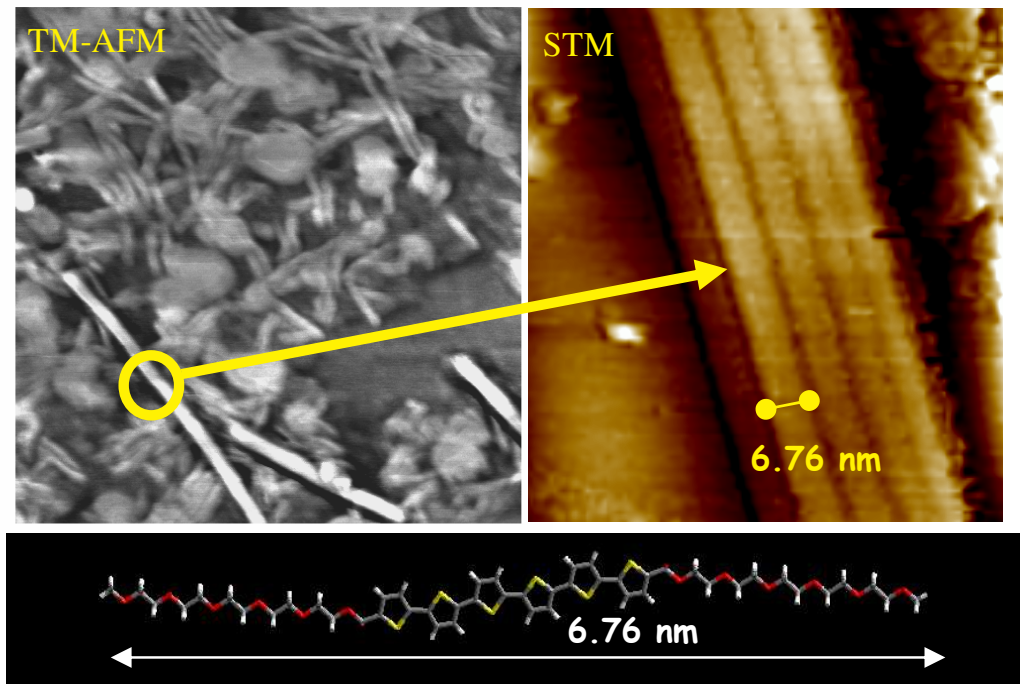

Fig. 5 Organisation supramoléculaire d'oligothiophénes (aimablement transmis par R. Lazzaroni, Univ. de Mons)

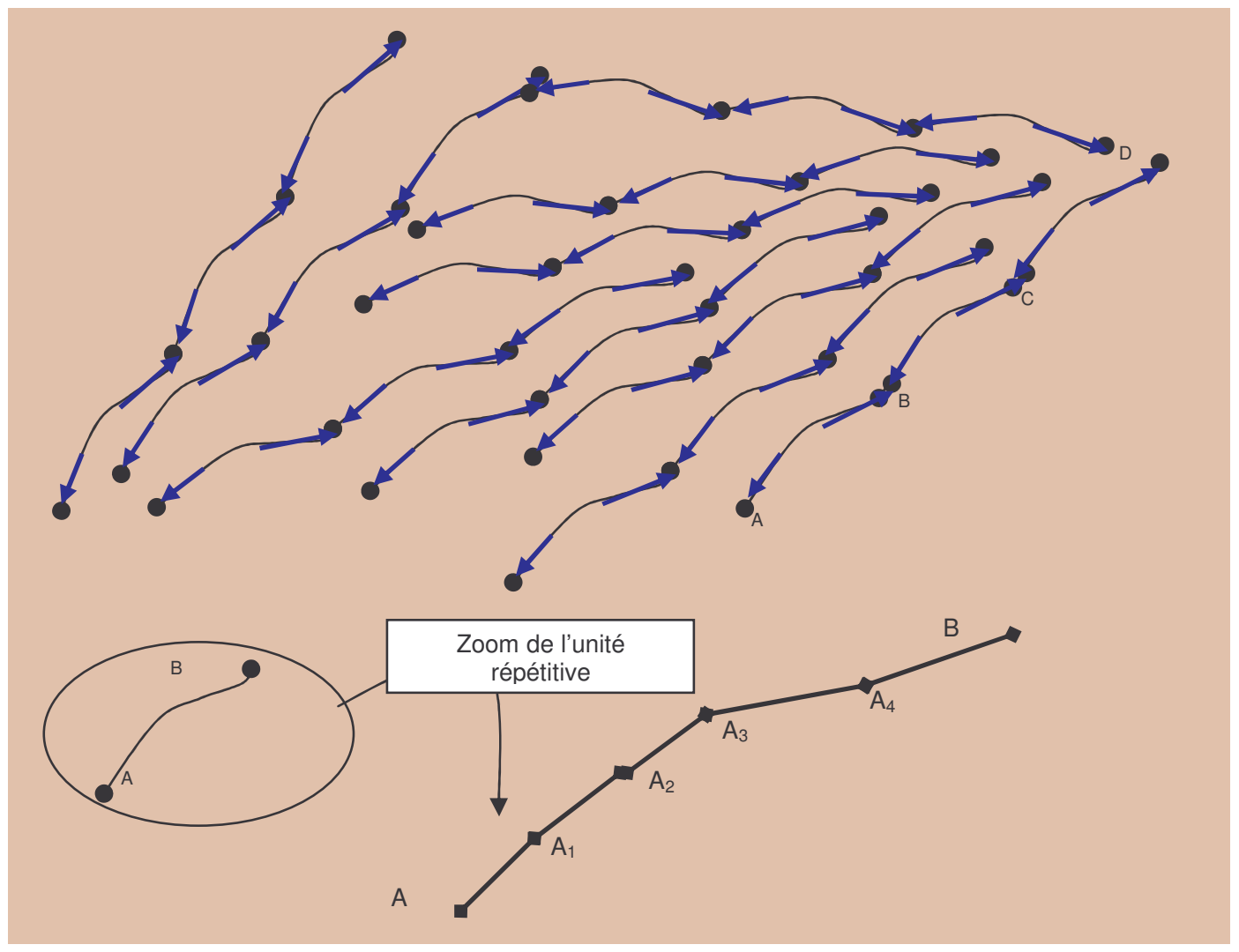

Fig. 6 Le monomère $A B$ représenté dans la bulle est en fait constitué de 5 molécules mères $A_{1}, A_{1} A_{2} ; A_{2} A_{3}, A_{3} A_{4}$, et $A_{4} B$, toutes identiques entre elles, mais ne se présentant pas avec les mêmes orientations spatiales. Ensuite, 3 monomères identiques $A B, B C$, et $C D$ sont greffés entre eux pour constituer l'oligomère $\mathrm{AD}$ à 15 mères. Chacune des chaînes de type $\mathrm{AB}$ peut alors présenter des orientations diverses dans l'espace, offrant l'image d'un milieu apparemment désorganisé. 

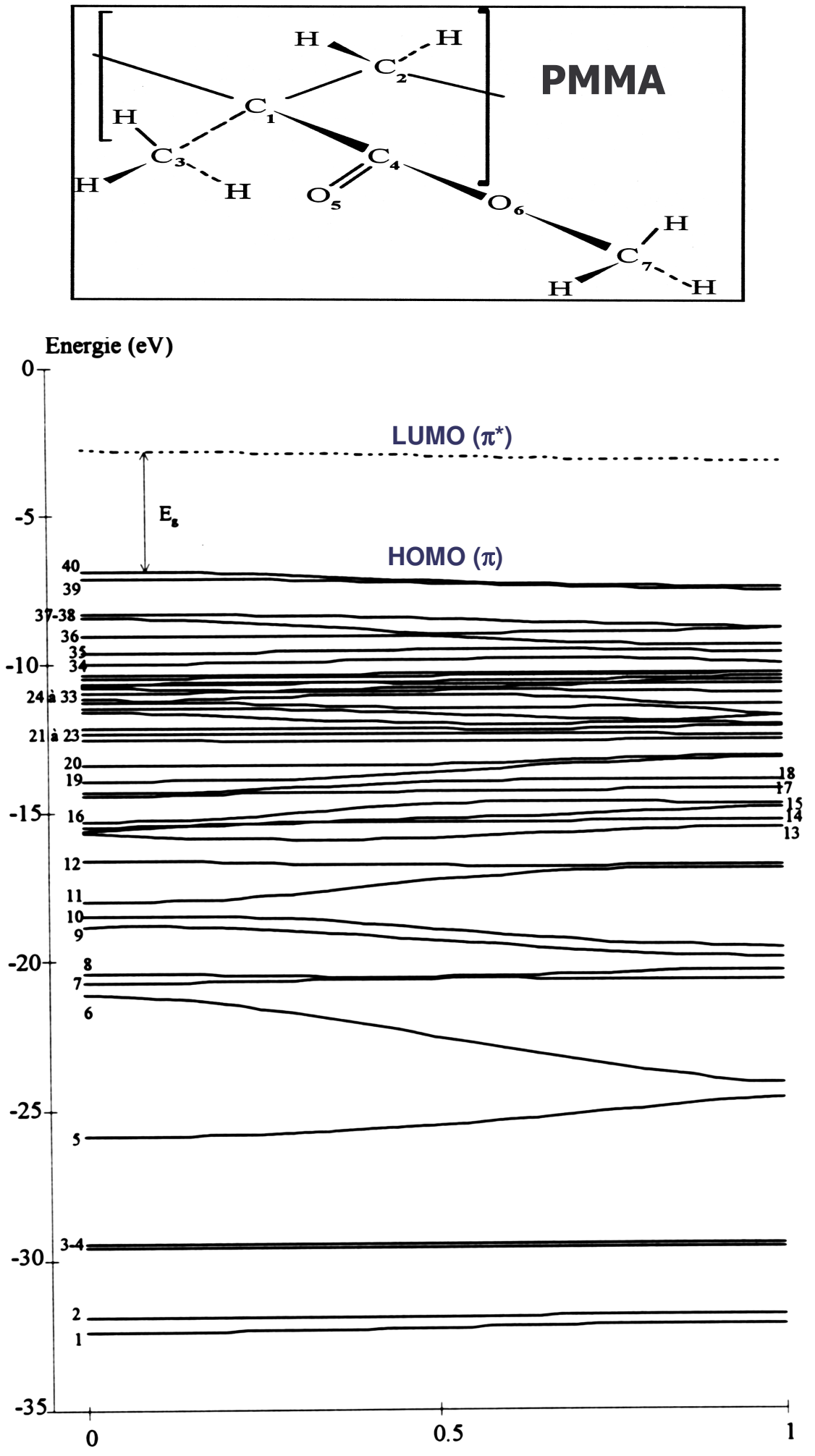

Fig. 7 Première zone de Brillouin du PMMA. Le niveau de Fermi est placé exactement au milieu de la bande interdite Eg définie par le domaine compris entre le niveau HOMO et le niveau LUMO $; \mathrm{E}_{\mathrm{g}}=5,5 \mathrm{eV}$. 


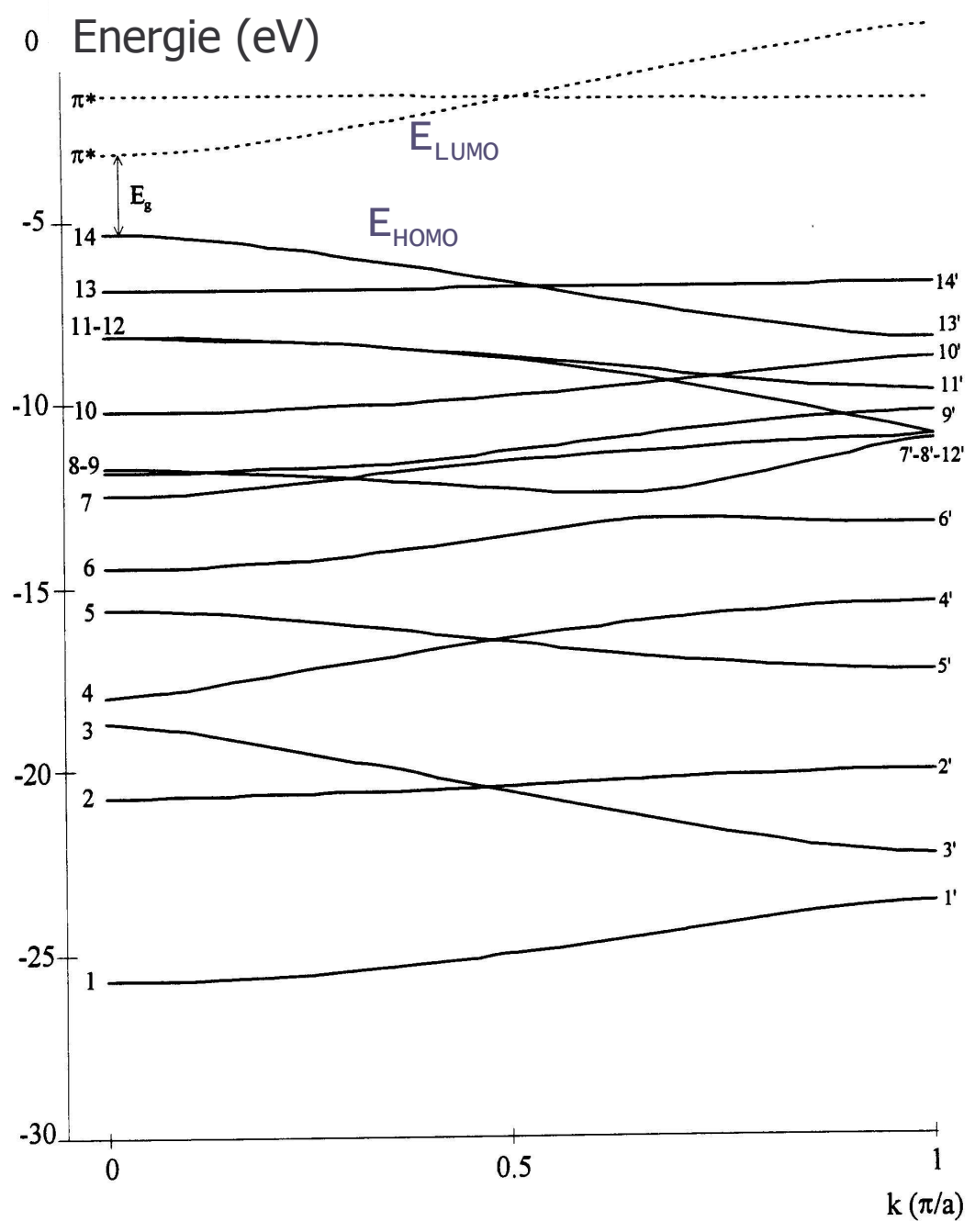

Fig. 8 Structure de bande du PPP. La bande HOMO est placée à $-7,57 \mathrm{eV}$, et la bande LUMO à $-4,71 \mathrm{eV}$, définissant une bande interdite $\mathrm{E}_{\mathrm{g}}=\mathrm{E}_{\mathrm{LUMO}}-\mathrm{E}_{\text {HОМO }}=2,85 \mathrm{eV}$. 


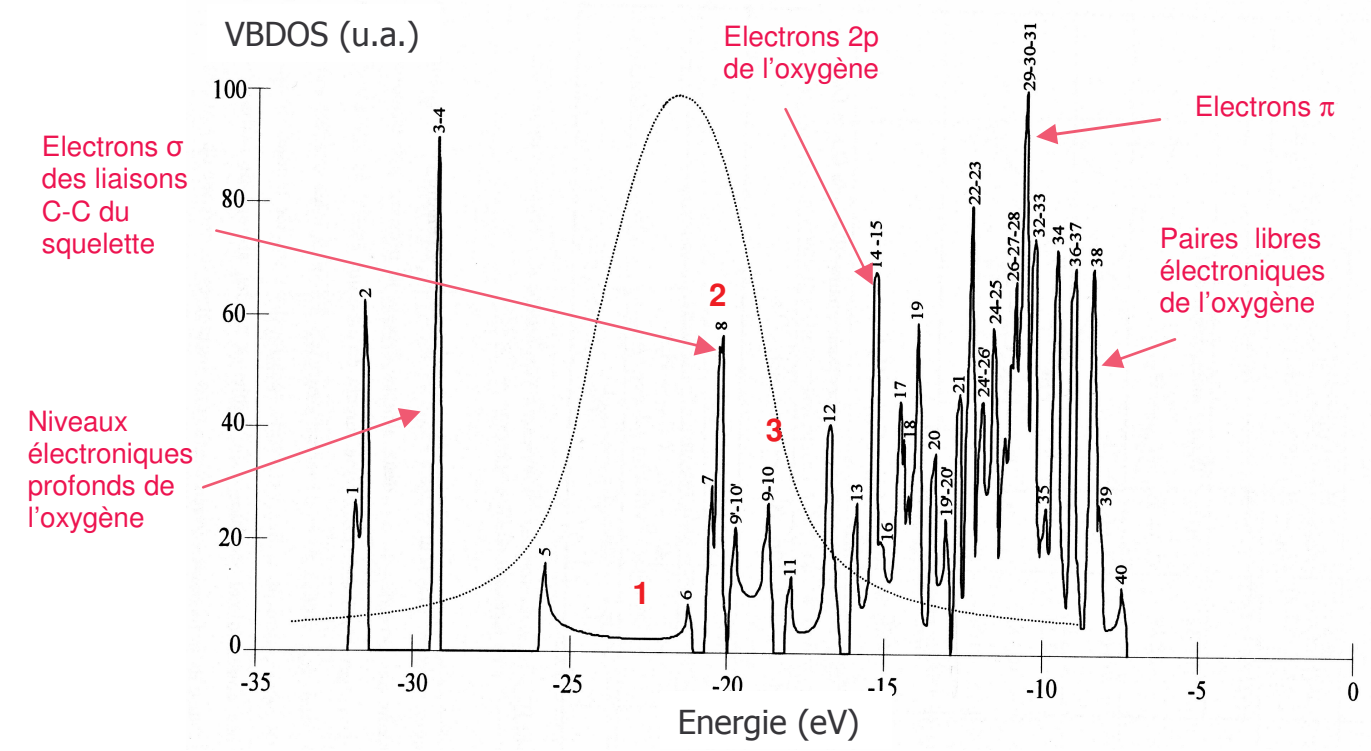

Fig. 9 VBDOS du PMMA. Chaque bande regroupe une catégorie d'électrons qui, contribuant à l'établissement d'une liaison, disposent de la même énergie parce qu'ils sont placés sur la même orbitale. A titre d'exemple, 5 ont été reportées. La courbe en cloche, à profil de Lorentzienne, est la courbe de résonance plasmon centrée à la fréquence $\omega_{\mathrm{rp}}=21,5 \mathrm{eV}$.

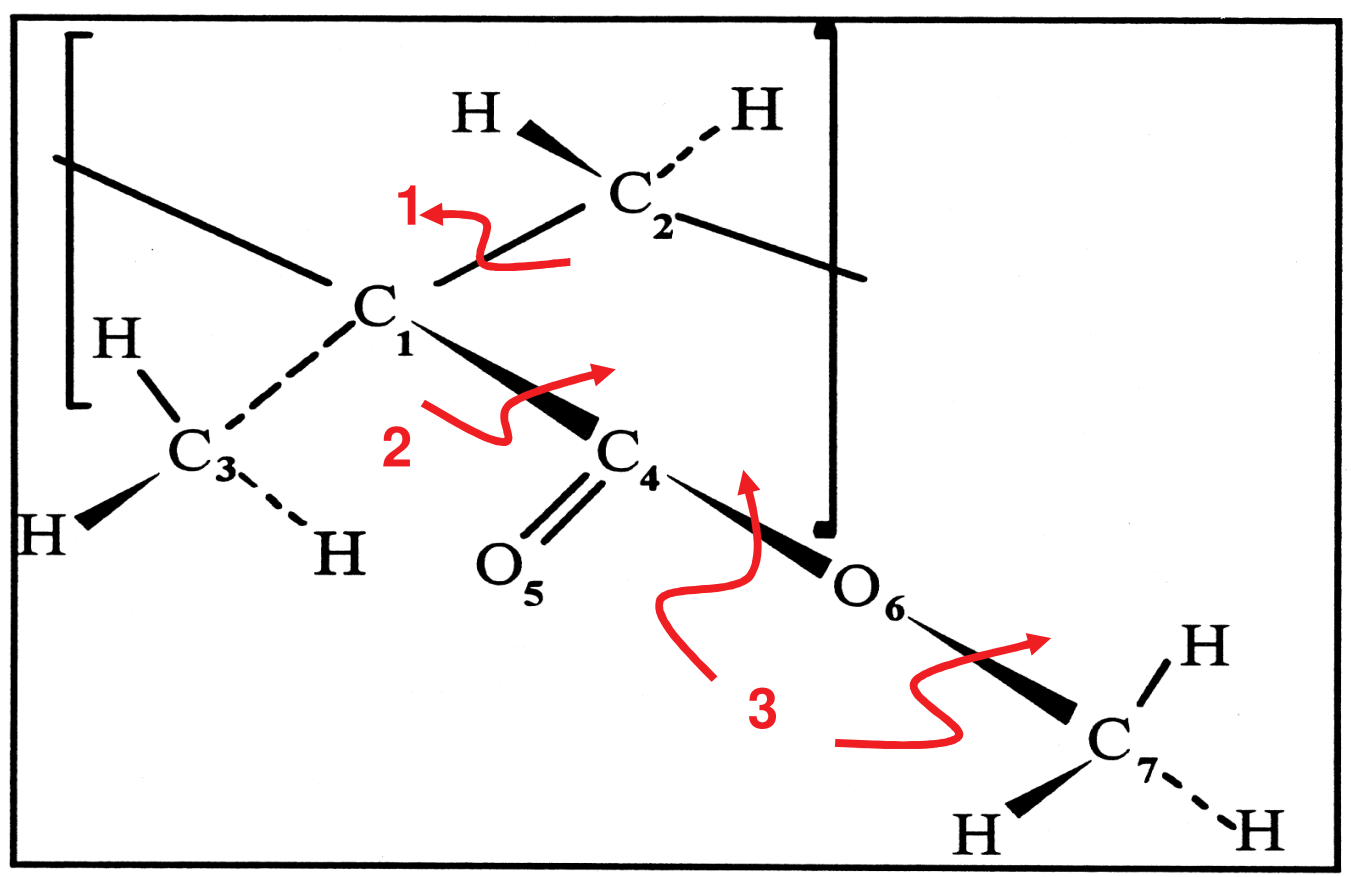

Fig. 10 Illustration de l'endommagement sélectif du PMMA par le phénomène de résonance plasmon lors du passage d'une particule énergétique. Les ruptures des liaisons répertoriées 1, 2 et 3 correspondent aux bandes portant les mêmes chiffres de repérages dans la représentation de la VBDOS de la figure 9. 


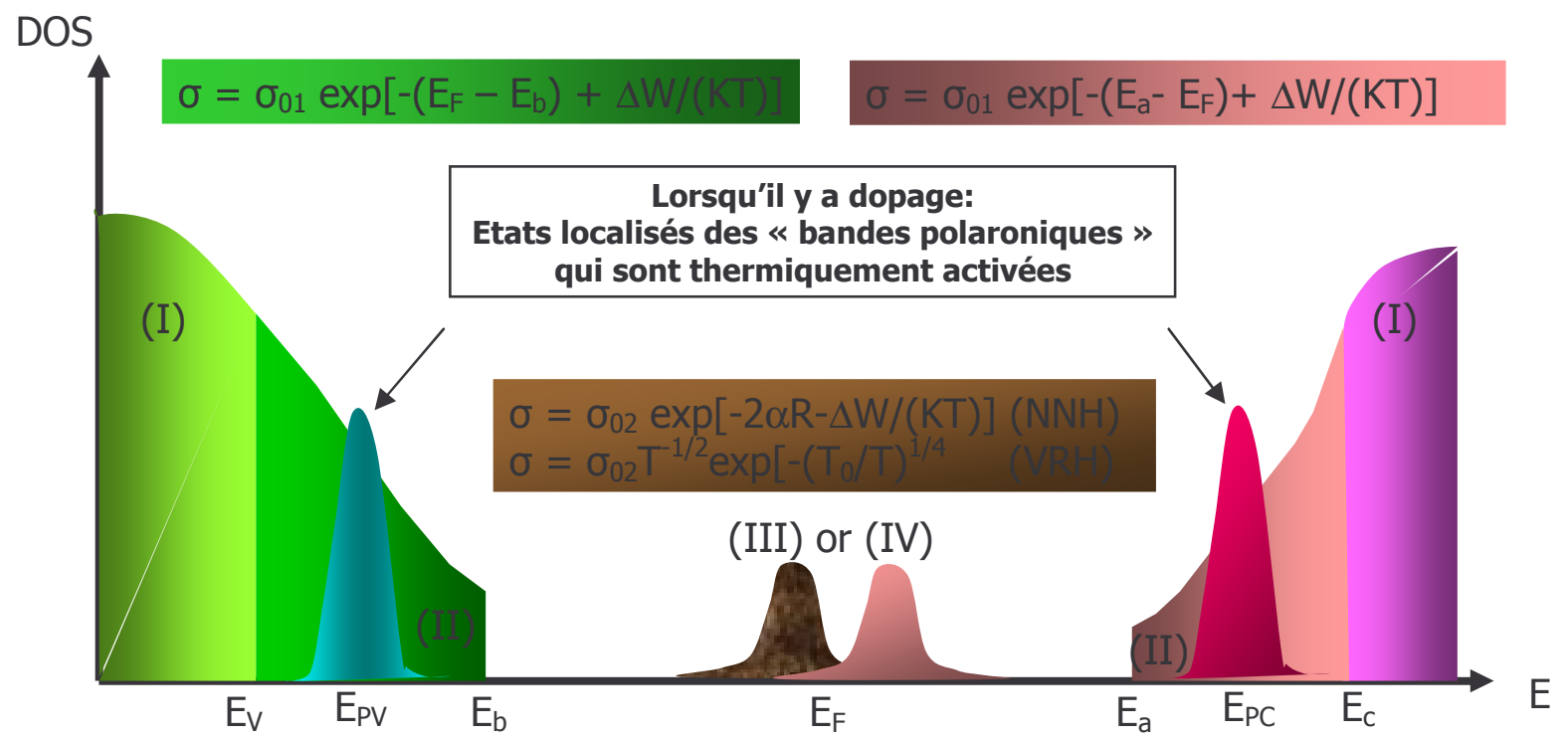

Fig. 11 Différents modèles de conduction électronique dans les milieux organiques suivant la position des états d'énergie dans la structure de bande.

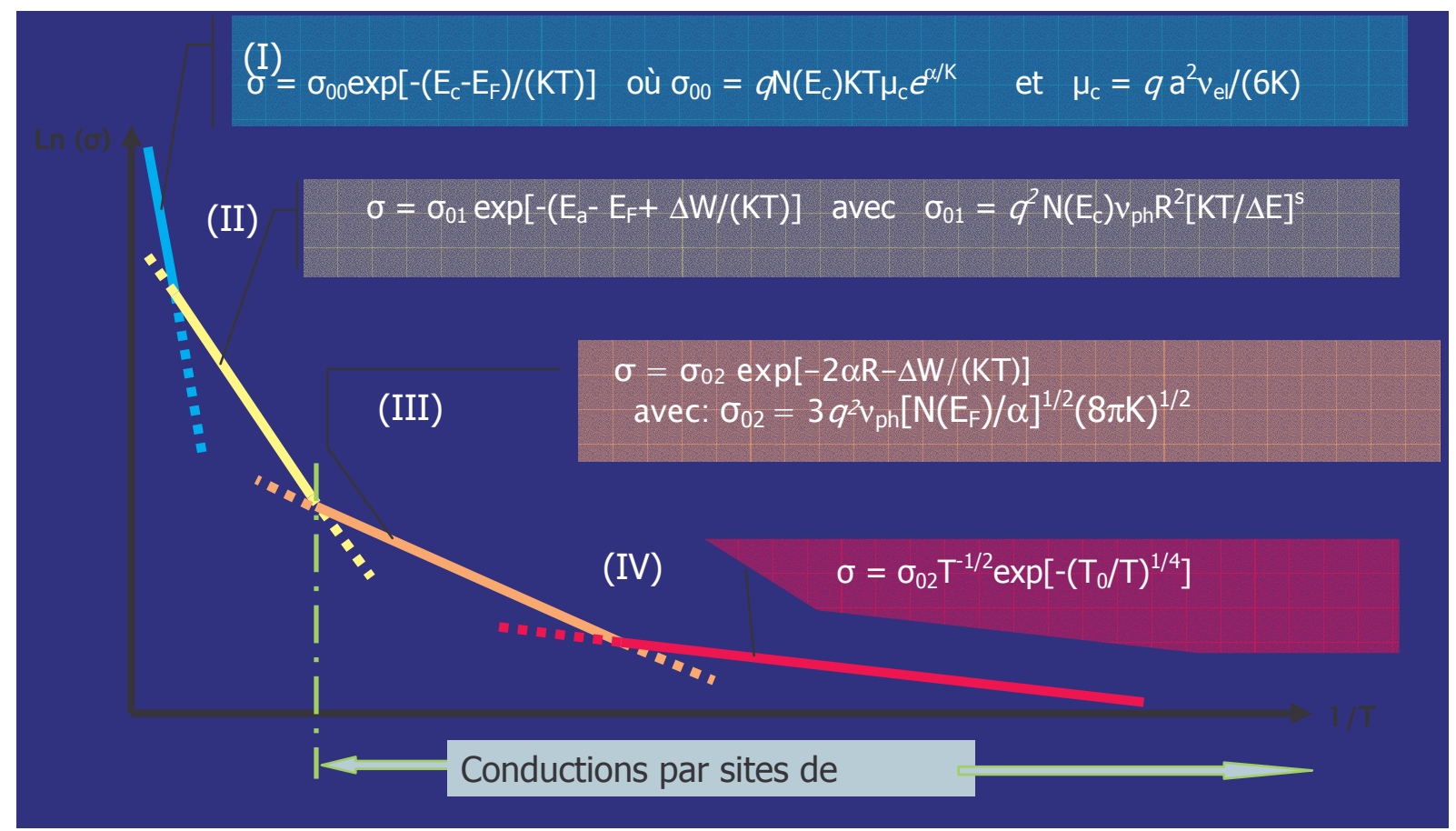

Fig. 12 Schématisation de la représentation des modèles de conduction électronique continue en fonction de 1/T. 


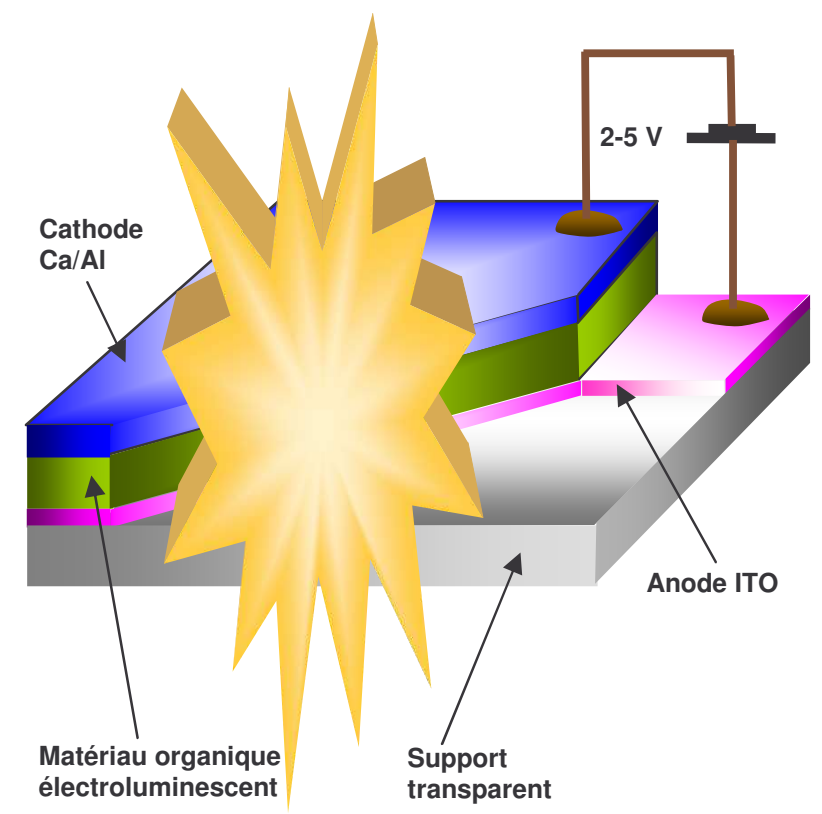

Fi. 13 Schéma de principe d'une diode organique électroluminescente en fonctionnement dans sa configuration élémentaire.

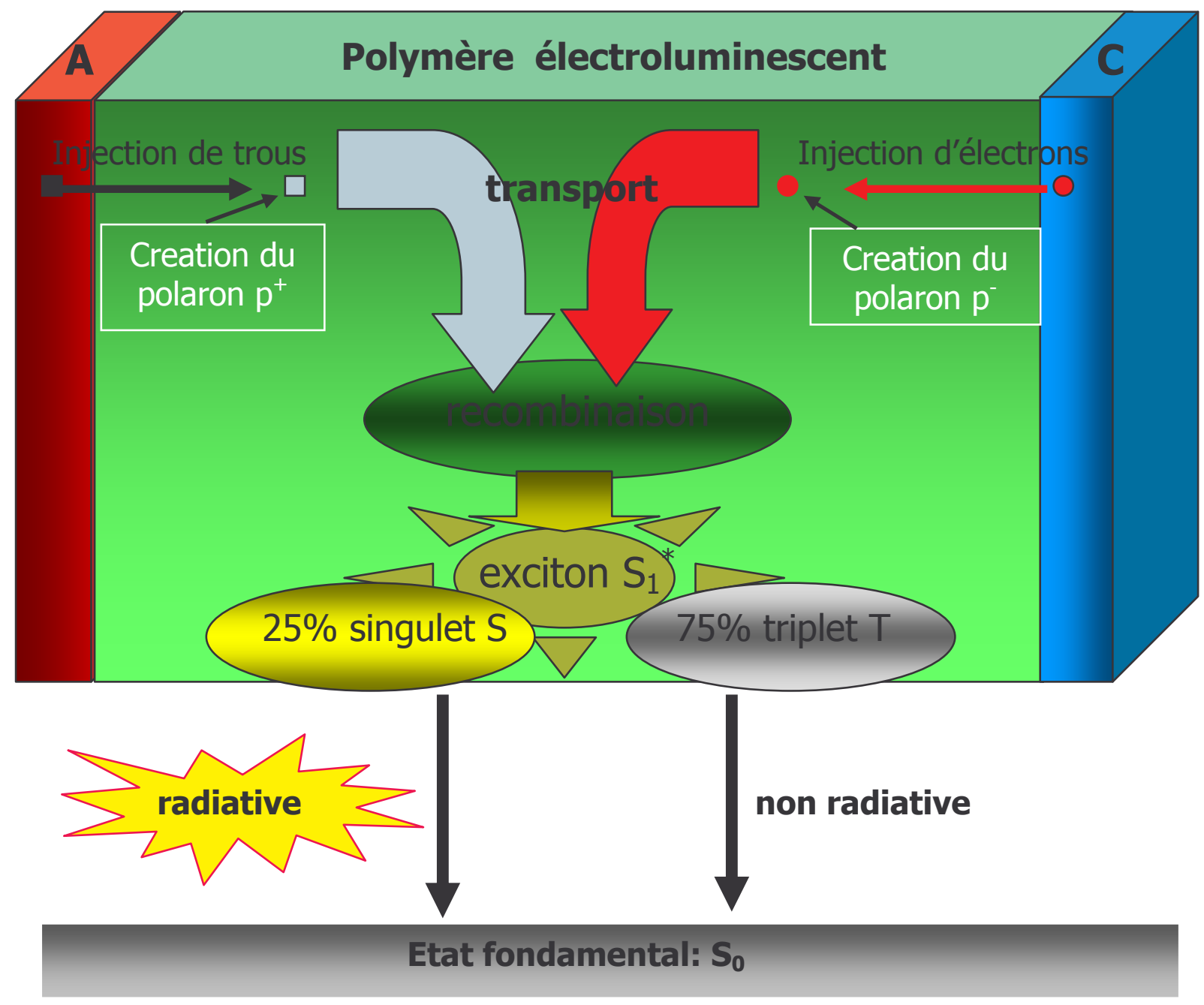

Fig. 14 Schématisation du mécanisme d'électroluminescence dans une OLED ou une PLED. 

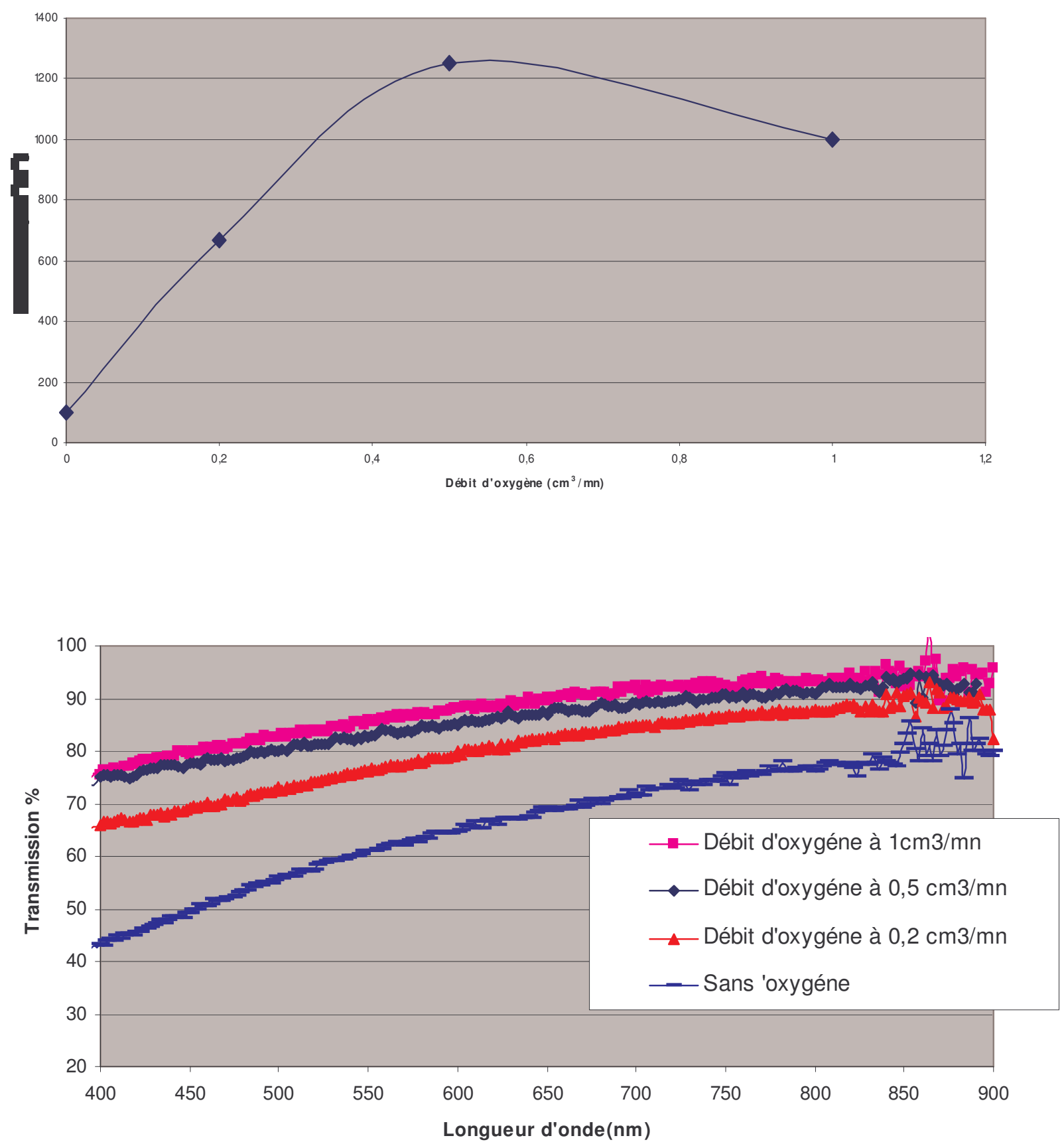

Fig. 15 Conductivité électrique et transmission optique d'ITO déposé par IBAD sur CR39 avec des flux variables en oxygène. Un recuit thermique améliore encore la transparence. Le meilleur compromis se situe autour d'un flux de $0,2 \mathrm{~cm}^{3} . \mathrm{s}^{-1}$ pour lequel on dépasse toujours $80 \%$ de transmission optique (B. Lucas, W. Rammal, XLIM/UMOP/CNRS) 


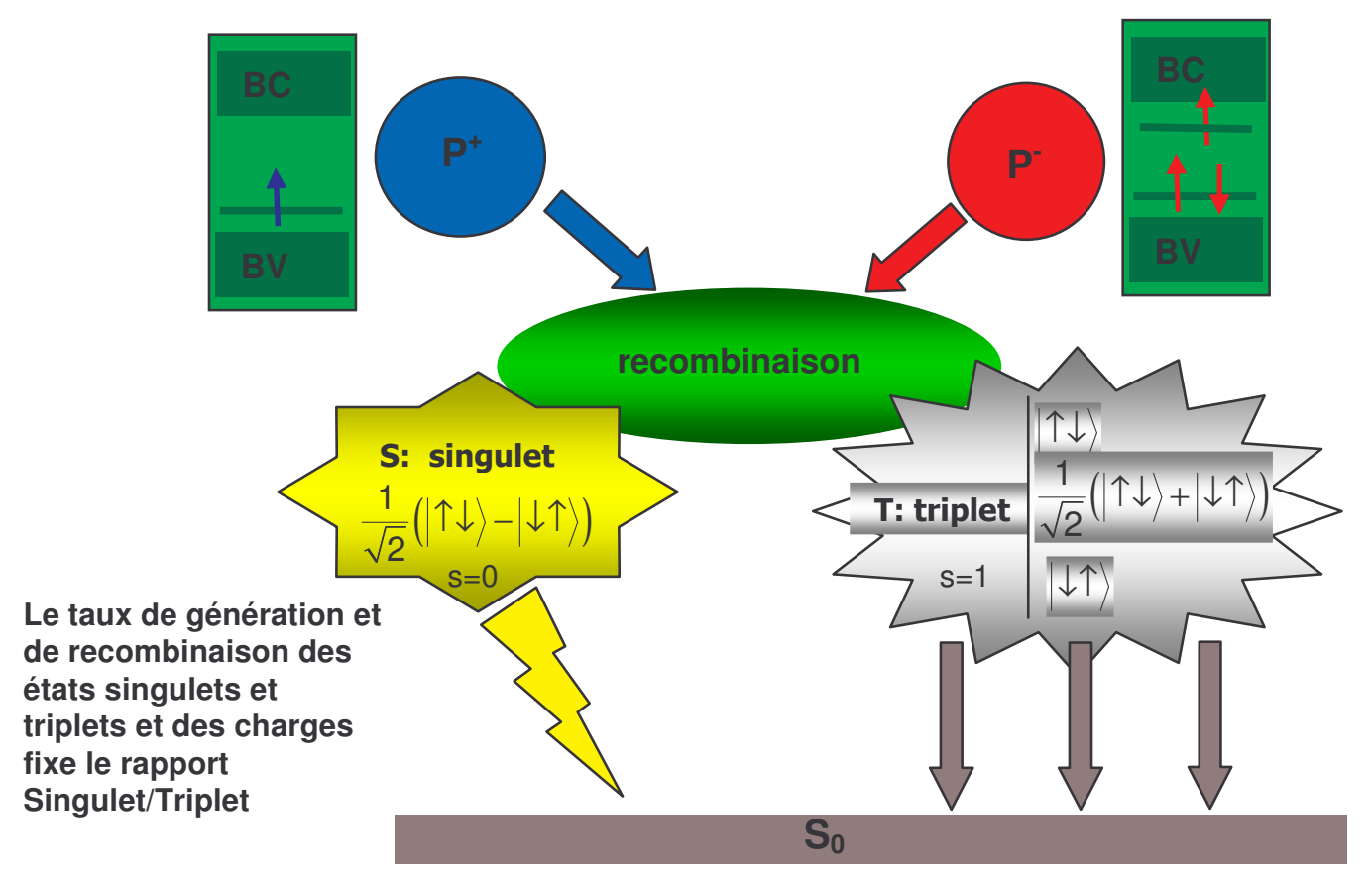

Si les probabilités de formation de $\mathrm{S}$ et de $\mathrm{T}$ sont identiques, alors:

\section{$\mathrm{S} / \mathrm{T}=1 / 3$}

Fig. 16 Compte tenu des états de spin des états polaroniques $\mathbf{p}^{+}$et $\mathbf{p}^{-}$, il existe trois fois plus d'états triplets que singulet. Le taux de recombinaison radiatif $\mathrm{r}_{\mathrm{st}}$ est donc $\mathrm{r}_{\mathrm{st}}=\mathrm{S} /(\mathrm{S}+\mathrm{T})=0,25$ au maximum par molécule.
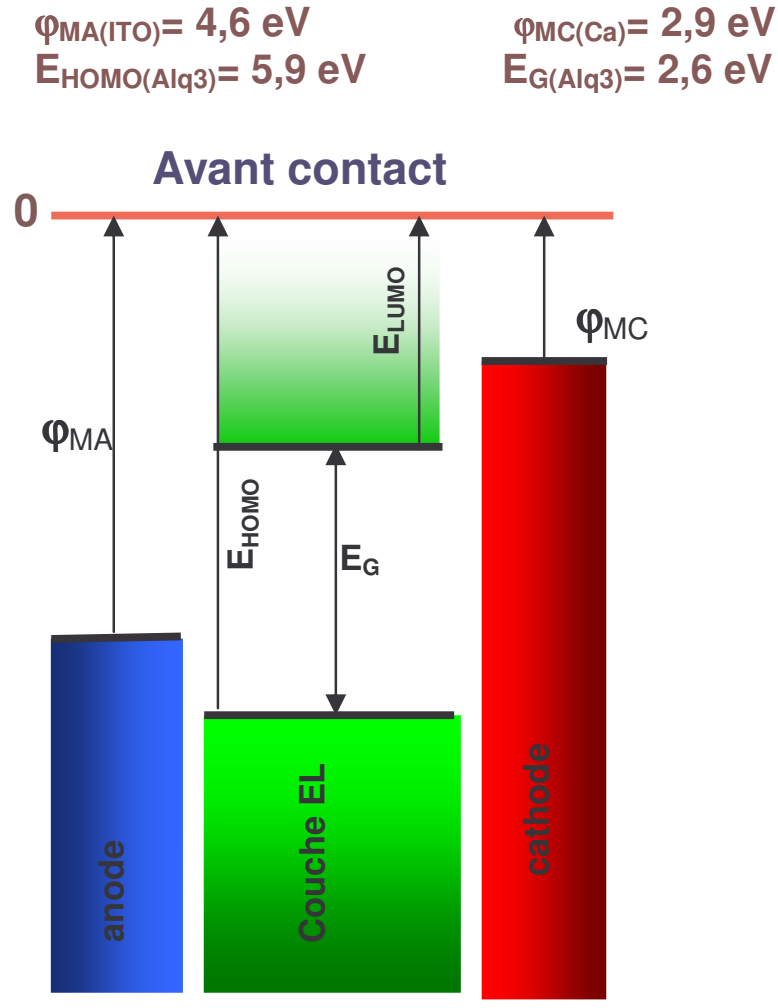

\section{Après contact}

-Alignement des niveaux de Fermi

-Pas de courbure de bande en l'absence de charge dans la couche EL

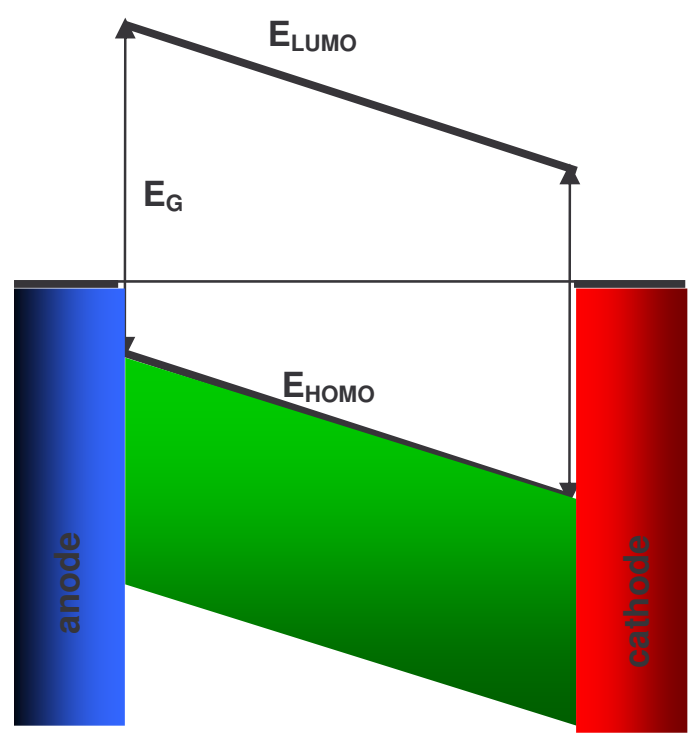

Fig. 17 Diagramme en énergie dans une OLED à base d'Alq ${ }_{3}$ avant et après contact sans polarisation. 


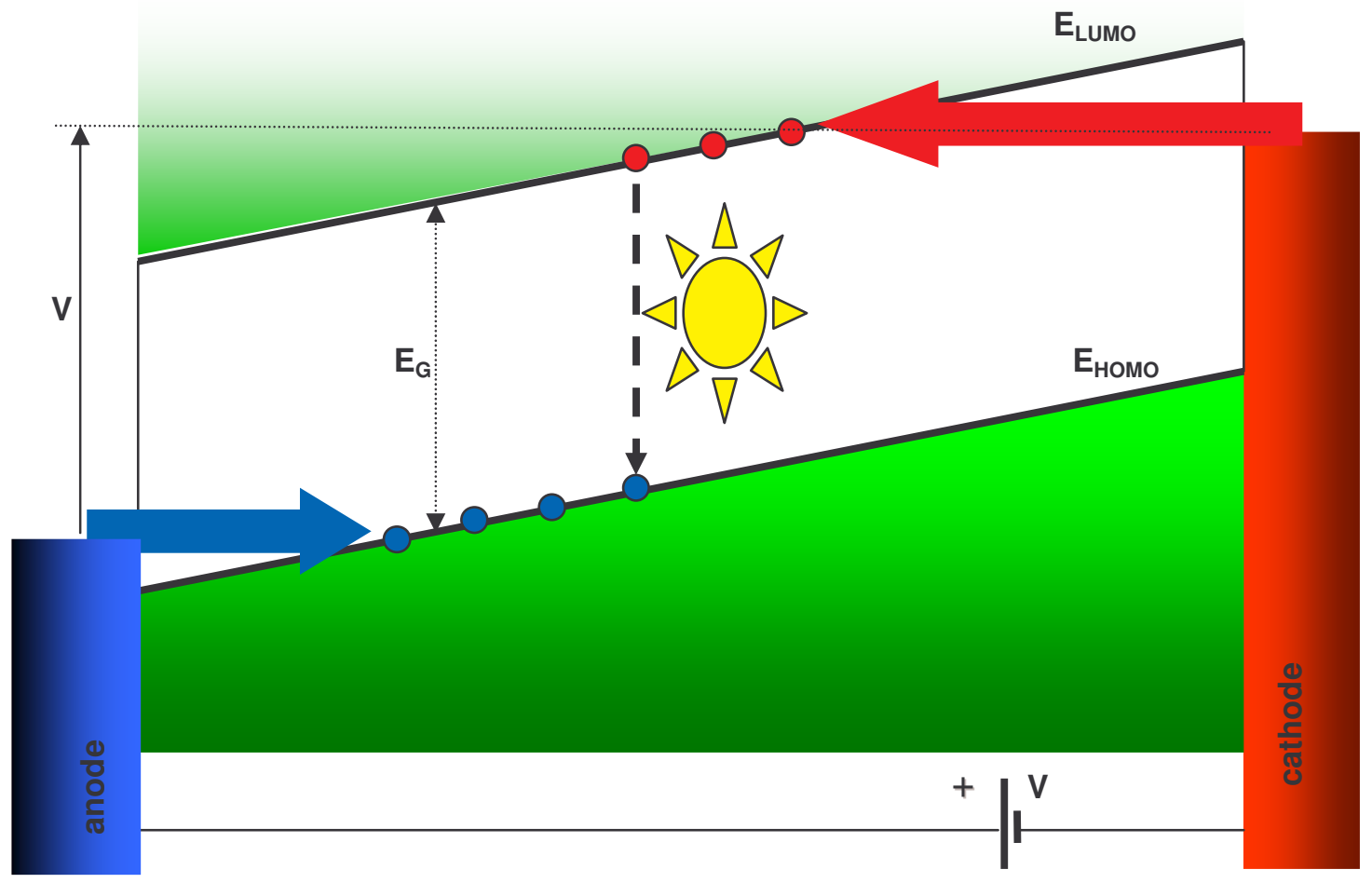

Fig. 18 Diagramme en énergie sous polarisation. Les électrons injectés à la cathode et les trous injectés à l'anode traversent le milieu électroluminescent sous forme polaronique, et se recombinent dans le milieu organique pour donner lieu à la luminescence.

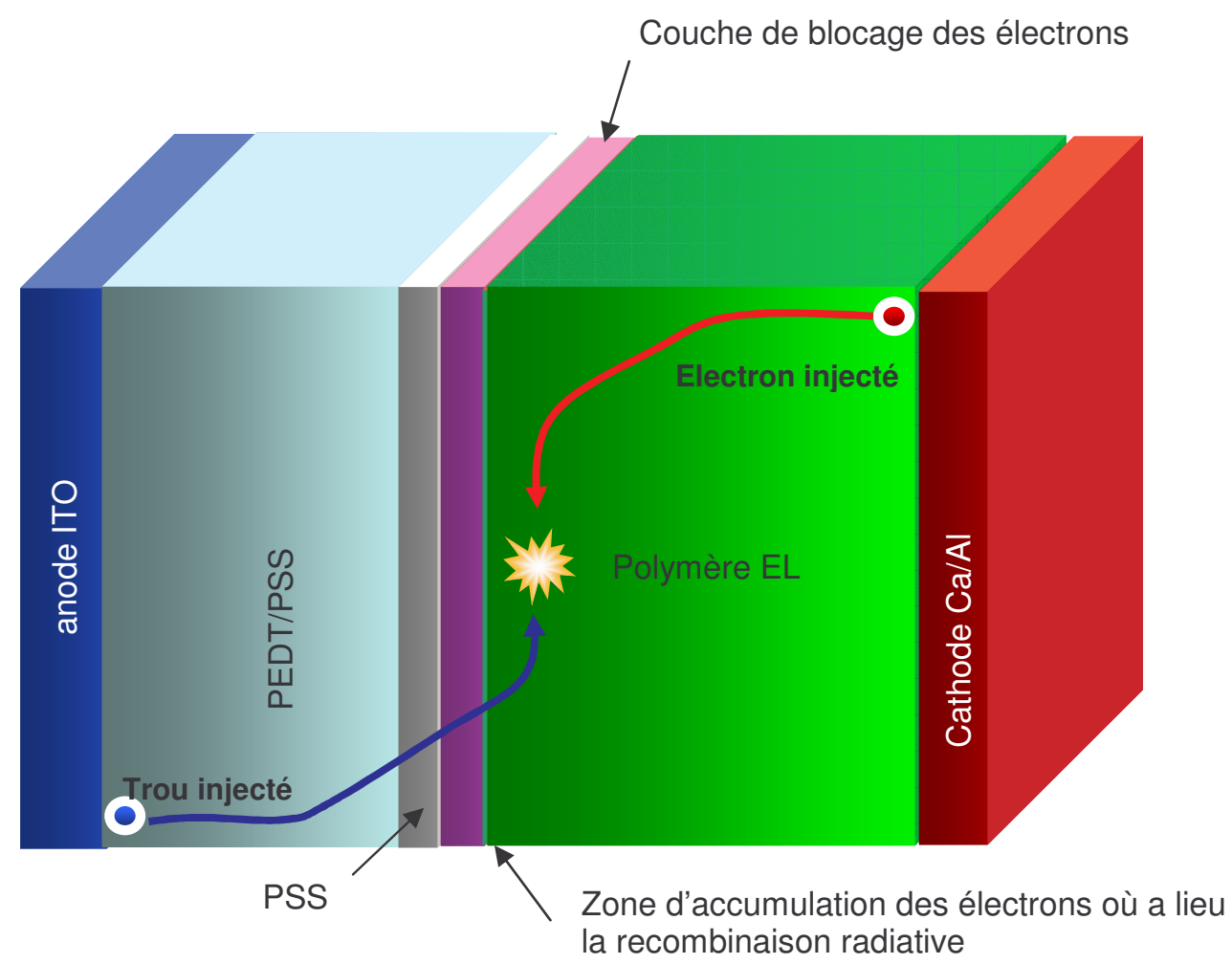

Fig. 19 Structure actuelle d'une PLED multicouche. Le polymère électroluminescent sert également au transport des électrons. Une couche de PEDT facilite le transport des trous, alors qu'une fine couche de PSS permet l'injection des trous par effet tunnel. Une couche de blocage des électrons permet de les accumuler dans le polymère électroluminescent (EL) afin d'augmenter la probabilité de recombinaison radiative dans ce dernier et d'améliorer le rendement. 


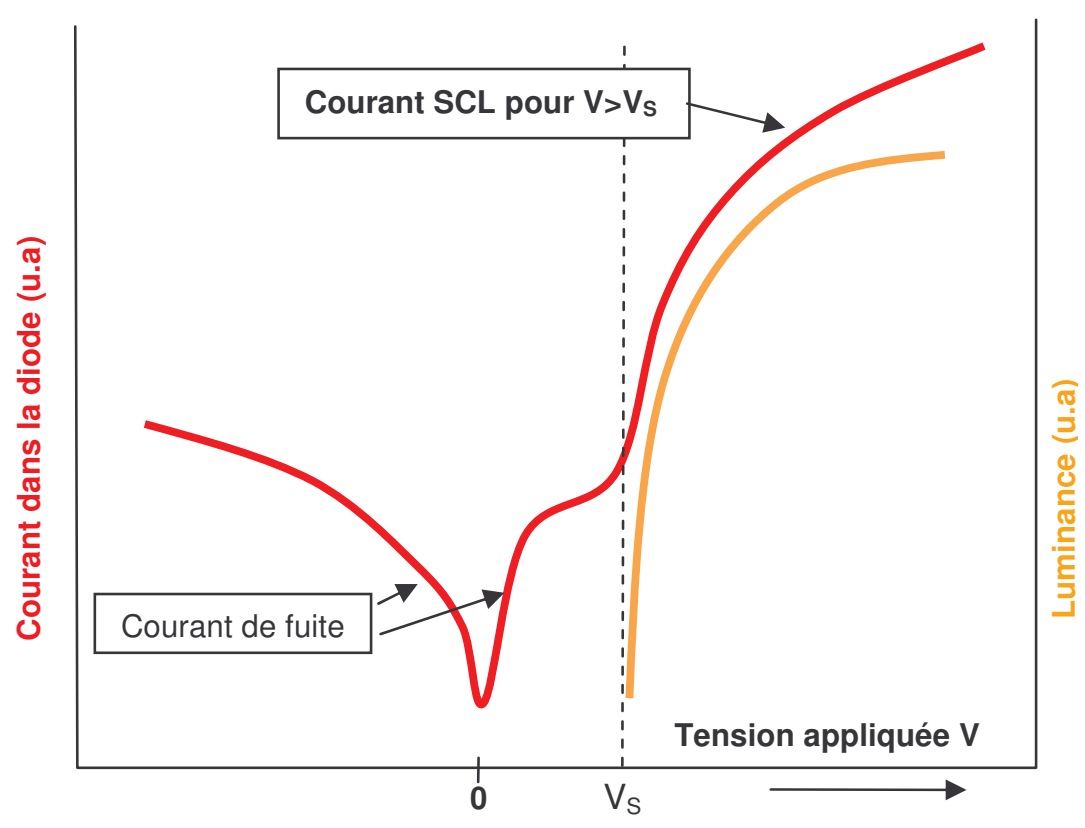

Fig. 20 Allure générale des caractéristiques courant - tension, et luminance - tension dans une LED organique. Au-delà de la tension de seuil VS, les deux courbes ont sensiblement la même allure jusqu'au maximum de l'émission. VS se situe en général entre 1 et 3 V. Les courants peuvent atteindre 1 A.cm-2, et la luminance 10 Cd.cm-2. Les meilleures efficacités lumineuses sont de l'ordre de 2,5 Cd.A-1.
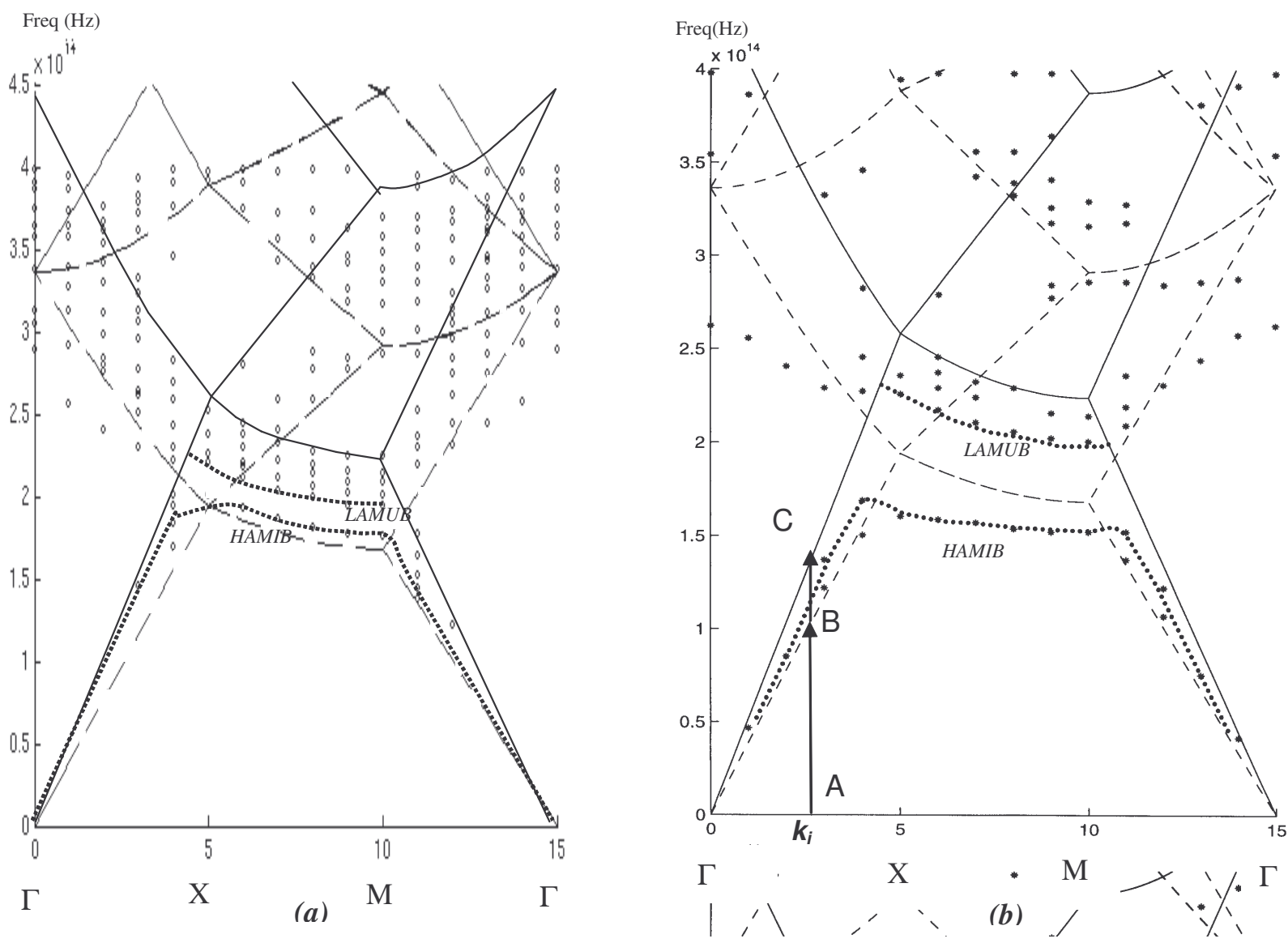

Fig. 21 Structures de bande d'un réseau triangulaire en polarisation TE (a) et TM (b). Une bande interdite existe dans les deux types de polarisations, mais est bien plus étroite en polarisation TE qu'en TM. Pour le vecteur d'onde $\mathbf{k}_{\mathbf{i}}$, les longueurs d'onde comprises entre A et B se propagent dans le substrat, entre B et C dans le matériau organique structuré, et au dessus de $\mathrm{C}$ dans le surstrat. 


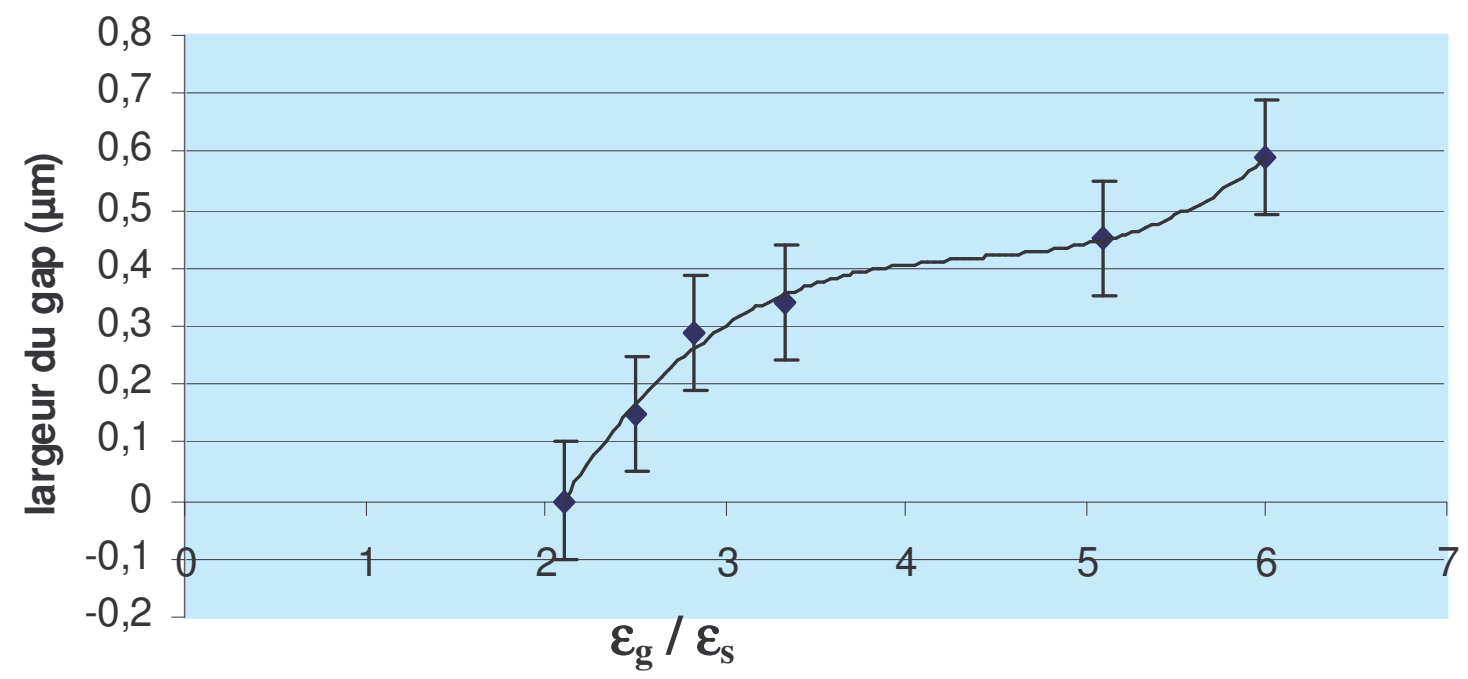

Fig. 22 Influence du contraste de permittivité sur la largeur de la bande interdite dans le cas d'un réseau triangulaire. Ce n'est qu'à partir d'un contraste au moins égal à 2,13 que la bande interdite s'ouvre.

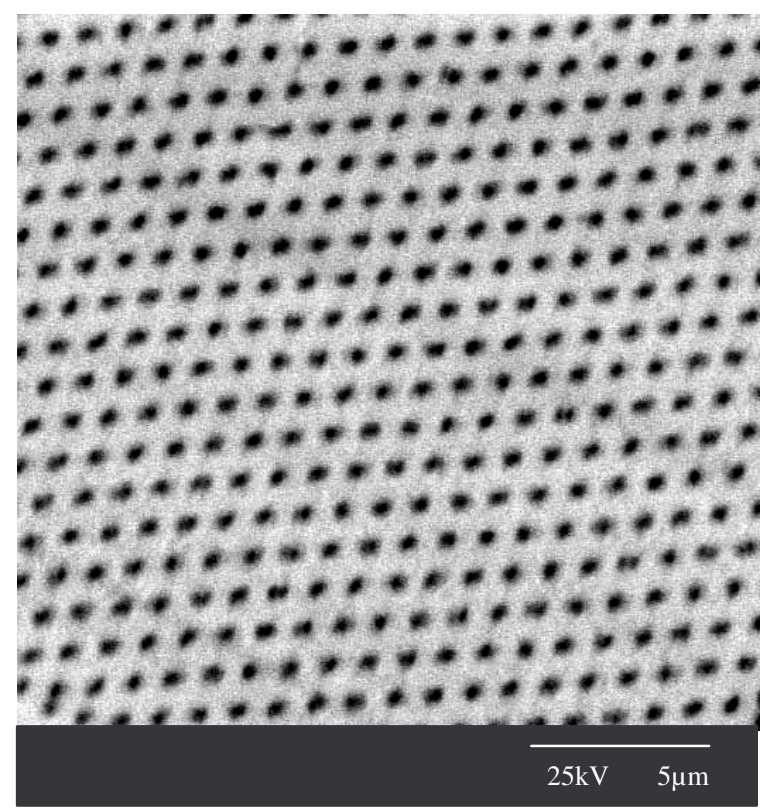

(a)

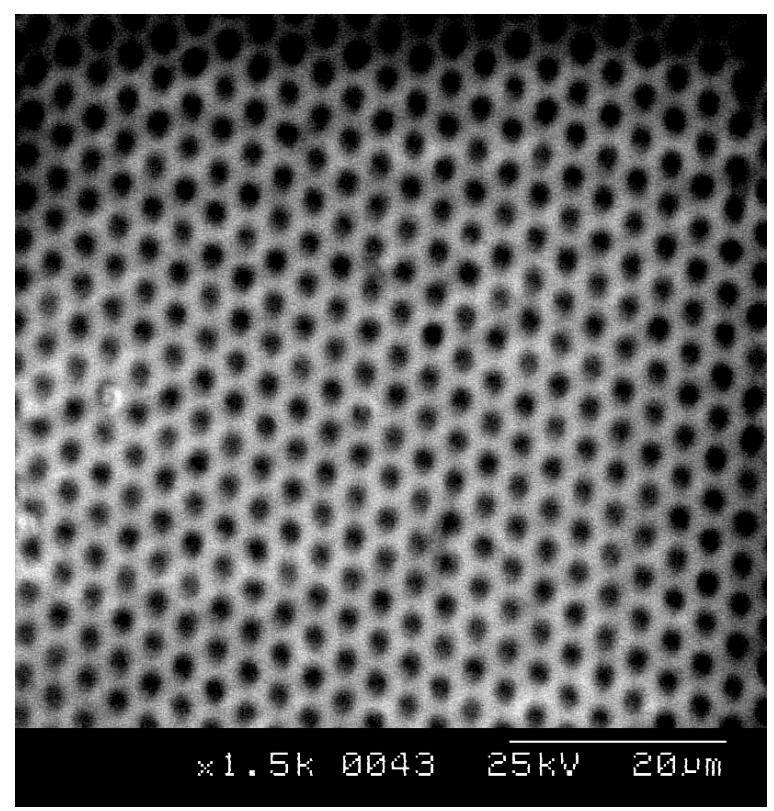

(b)

Fig. 23 Réseaux triangulaires gravés dans le CR39 par FIB. (a) vue en microscopie ionique à balayage d'un réseau à la période de $2 \mu$ m, de trous de $400 \mathrm{~nm}$ de diamètre et une profondeur de l'ordre de $200 \mathrm{~nm}$. (b) vue au MEB, après une préparation spéciale d'un réseau d'une période de $5 \mu \mathrm{m}$, de trous de diamètre $1,2 \mu \mathrm{m}$ pour une profondeur de gravure de $400 \mathrm{~nm}$. 